ARTIGO DE REVISÃO

JULIA MACHADO KHOURY JOÃO PEDRO SOUSA DRUMOND

LETÍCIA LOPES DE CARVALHO E SILVA

MARIANE DA SILVA MELO

ANANDA ARAUJO TEIXEIRA

MAILA DE CASTRO LOURENÇO DAS NEVES

FREDERICO DUARTE GARCIÁ

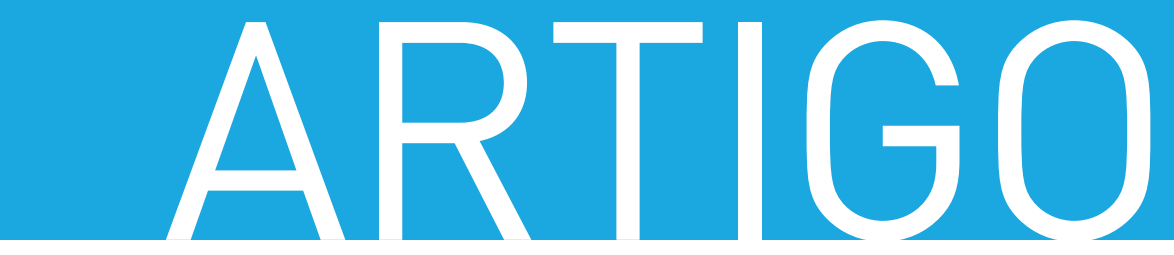

\title{
FATORES ASSOCIADOS À DEPENDÊNCIA DE SMARTPHONE: UMA REVISÃO DA LITERATURA
}

\section{FACTORS ASSOCIATED TO SMARTPHONE ADDICTION: A LITERATURE REVIEW}

\section{Resumo}

Conhecer melhor o que se chama de dependência de smartphone (DS) é importante para a prática clínica. Nos últimos 10 anos, um corpo de evidências clínicas e epidemiológicas foi produzido em vários países. Esses estudos apontam fatores sociodemográficos, hábitos de vida, transtornos psiquiátricos e traços de personalidade associados à DS. Contudo, não há estudos de revisão que sintetizem os resultados. Objetivamos revisar a literatura sobre os fatores associados à DS. Foi realizada uma revisão bibliográfica sistemática nas bases de dados PubMed e SciELO. Restringimos a busca aos artigos publicados nos últimos 10 anos. Excluímos revisões de literatura, estudos pré-clínicos e opiniões de especialistas. Na fase final, selecionamos 42 artigos. Os fatores sociodemográficos associados à DS foram sexo feminino, idade entre $18 \mathrm{e}$ 25 anos e alta renda familiar. Os hábitos de vida foram cronótipo noturno, baixa atividade física e sedentarismo. Os conteúdos mais associados à DS foram as redes sociais. As síndromes de dependência mais associadas à DS foram as relacionadas ao álcool e tecnológicas. Os transtornos e/ou sintomas psiquiátricos associados foram os transtornos depressivos e ansiosos, o estresse e a insônia. Por fim, os traços de personalidade associados foram alta impulsividade, alta agressividade, baixa autoestima, personalidade do tipo A e personalidade multitarefa. Existe um corpo de evidências que mostra semelhanças entre os achados epidemiológicos encontrados para a DS e para outras formas de dependência. Mais estudos são necessários para aprofundar o nosso conhecimento sobre esse tema.

Palavras-chave: dependências comportamentais.

\section{Abstract}

Improving our knowledge of what has been referred to as smartphone addiction (SA) is extremely important for clinical practice. In the past 10 years, an increasing body of clinical and epidemiological evidence has been produced in several countries. These studies point to sociodemographic factors, life habits, psychiatric disorders, and personality traits in association with SA. However, no review studies have been conducted to synthesize the findings. The objective of this study was to review the literature currently available on factors associated with SA. A systematic literature review was carried out by searching the PubMed and SciELO databases. We focused on papers published in the last 10 years; literature reviews, pre-clinical studies and expert opinions were excluded. In the final phase, 42 articles were selected. The sociodemographic factors associated with SA were female sex, age between 18 and 25 years and high household income. The associated lifestyle habits were nocturnal chronotype, reduced physical activity and sedentary lifestyle. The contents most strongly associated with SA were social networks. The dependency syndromes most strongly associated with SA were alcohol and technological addictions. The psychiatric disorders/symptoms associated were depressive and anxiety disorders, stress and insomnia. Finally, the associated personality traits were high impulsiveness, high aggressiveness, low self-esteem, type A personality and multitasking. There is evidence pointing to similarities in the epidemiological data found for SA and for other dependencies. More studies are needed to deepen our knowledge of this topic.

Keywords: Smartphone addiction, technological addictions, behavioral addictions. 


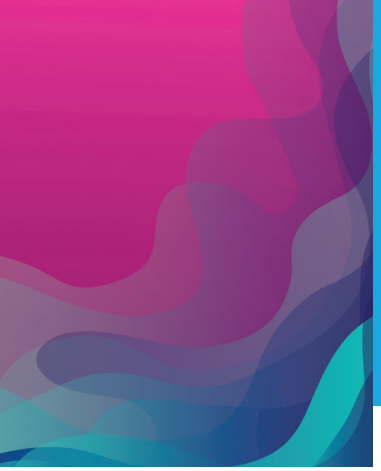

\section{INTRODUÇÃO}

Os smartphones invadiram a nossa sociedade de forma intensa e quase onipresente, modificando o comportamento social, afetivo e interpessoal dos indivíduos ${ }^{1-3}$. Apesar dos inquestionáveis benefícios providos pelas tecnologias móveis, como o acesso portátil e fácil à informação, o uso disfuncional dos smartphones tem sido associado a prejuízos como a redução do desempenho acadêmico e laboral ${ }^{4,5}$, o aumento dos transtornos e de sintomas do sono ${ }^{6}$ e o aumento da prevalência de acidentes automobilísticos ${ }^{7}$. Além desses danos já comprovados, alguns autores evocam potenciais prejuízos de longo prazo em indivíduos que fazem um uso disfuncional dos smartphones ou que apresentam sintomas semelhantes aos das dependências quando fazem uso do aparelho.

A dependência de smartphone (DS) ainda não foi oficialmente incluída nas classificações nosológicas internacionais como um transtorno psiquiátrico. Contudo, nos últimos 10 anos, diversos estudos sugerem que certos padrões de comportamento resultantes do uso de smartphones assemelham-se àqueles observados em pacientes com dependências químicas e comportamentais ${ }^{8-13}$. Algumas dessas características em comum são:

1) uso do smartphone por um período maior do que o inicialmente previsto;

2) uso compulsivo e a dificuldade de controle de uso;

3) estreitamento do repertório;

4) continuidade de uso apesar dos prejuízos causados por ele;

5) necessidade deaumento progressivo da frequência e/ou intensidade do uso ${ }^{8-15}$.

Outros estudos encontraram uma associação entre a DS e fatores de risco sociodemográficos ${ }^{10,16-23}$, hábitos de vida13,24, transtornos psiquiátricos ${ }^{10,18,19,23,25-27}$ e traços de personalidade ${ }^{10,28-32}$ semelhantes aos observados em outras formas de dependências.

$\mathrm{O}$ estudo dos fatores associados à DS pode facilitar o estabelecimento de um paralelo entre esse constructo e outras formas de dependências, auxiliando no reconhecimento da DS como um transtorno psiquiátrico. Ademais, o estudo dos fatores associados pode orientar futuros estudos longitudinais para a identificação de fatores de risco e consequências da DS. A identificação de fatores de risco e consequências pode facilitar o desenvolvimento de estratégias específicas e mais eficazes para o diagnóstico, a prevenção, o tratamento e a reabilitação de pessoas apresentando esse padrão comportamental.

Neste artigo, realizamos uma revisão da literatura sobre os fatores associados à DS.

\section{MÉTodos}

Estratégia de busca

Realizamos uma revisão bibliográfica nas bases de dados PubMed e SciELO. A busca foi restrita ao idioma inglês e aos artigos publicados nos últimos 10 anos. Utilizamos os seguintes termos do Medical Subject Headings (MeSH): smartphone addiction; smartphone dependence; problematic smartphone use; mobile phone addiction; mobile phone dependence; problematic mobile phone use. Dois revisores pesquisaram de forma independente os títulos e resumos, lendo cuidadosamente artigos completos, quando relevantes.

\section{Critério de inclusão}

Foram incluídos estudos que avaliaram os fatores associados à DS por meio de análise univariada e/ou multivariada, e apenas estudos realizados em humanos e estudos clínicos. Foram excluídos estudos de revisão da literatura, estudos pré-clínicos, opiniões de especialistas e estudos não relacionados a fatores associados à DS.

\section{Extração e análise de dados}

Quando disponíveis, os seguintes dados foram recuperados dos estudos incluídos:

1) referência;

2) desenho do estudo;

3) transtorno principal abordado;

4) tamanho da amostra;

5) perfil dos participantes;

6) instrumentos utilizados;

7) objetivos;

8) resultados;

9) conclusões;

10) principais limitações. 
JULIA MACHADO KHOURY

JOÃO PEDRO SOUSA DRUMOND

LETÍCIA LOPES DE CARVALHO E SILVA

MARIANE DA SILVA MELO

ANANDA ARAUJO TEIXEIRA

MAILA DE CASTRO LOURENÇO DAS NEVES

FREDERICO DUARTE GARCIA
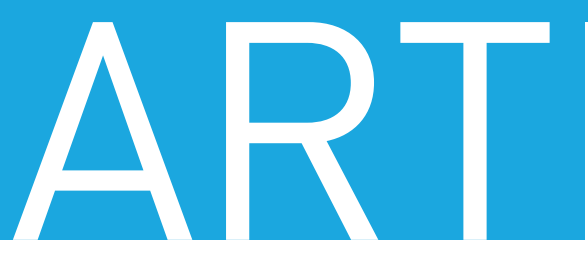

\section{Resultados}

O diagrama de fluxo (Figura 1) mostra os resultados quantitativos da revisão bibliográfica. Foram identificados 68 artigos na fase inicial, sendo que 13 foram excluídos porque avaliaram apenas o transtorno dependência de internet (DI), que é considerado um constructo diferente da DS. Oito artigos foram excluídos por analisarem exclusivamente os métodos de rastreamento da DS, a maioria deles relacionada à criação ou validação de instrumentos. Foram excluídos 22 artigos que analisaram apenas a eficácia de aplicativos desenvolvidos para o tratamento de outras doenças. Por fim, quatro artigos foram excluídos porque propunham apenas estratégias de prevenção e/ou tratamento para dependentes de smartphone. Na última etapa de seleção, incluímos 21 artigos das referências bibliográficas dos artigos elegíveis na etapa anterior. Todos os artigos incluídos na revisão bibliográfica apresentaram delineamento transversal.

Os principais resultados foram resumidos na Tabela 1. Nas seções seguintes, descrevemos os estudos de acordo com o grupo de fatores associados à DS.

\section{Fatores sociodemográficos}

Onze estudos encontraram associação positiva significativa entre o sexo feminino e a DS $10,16-23,28,52$. Os

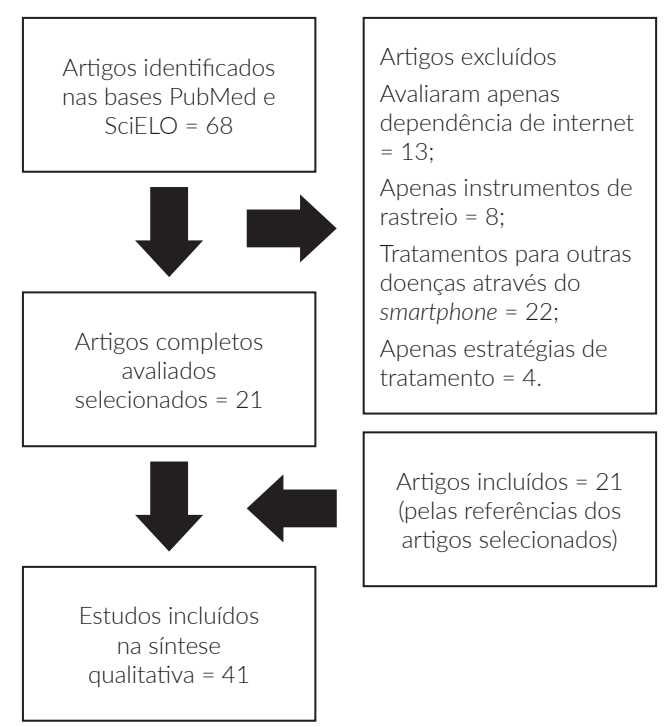

Figura 1 - Fluxo de seleção e inclusão de artigos para análise coeficientes betas variaram entre 0,10 e 1,46. Por outro lado, apenas um artigo encontrou associação positiva entre o sexo masculino e a DS 39 .

Cinco estudos encontraram uma associação positiva significativa entre as idades de 18 a 25 anos e a $\mathrm{DS}^{10,20,29,52,56}$. Os coeficientes betas variaram entre 0,17 e 0,24. Apenas um estudo encontrou uma associação positiva e significativa entre as idades de 15 a 16 anos e a DS, com um coeficiente beta de 0,5938.

Dois estudos realizados com estudantes universitários encontraram uma associação positiva e significativa entre a alta renda familiar mensal e a DS 24,25 . Por outro lado, apenas um estudo encontrou uma associação entre a baixa renda familiar mensal e a DS ${ }^{50}$. Finalmente, um estudo encontrou uma associação positiva e significativa entre o fato de ser matriculado em cursos das áreas humanas e a DS, com uma razão de chance de 2,1425.

Portanto, os principais fatores sociodemográficos associados à DS foram o sexo feminino, a idade jovem (18 a 25 anos) e a alta renda familiar mensal.

\section{Hábitos de vida}

Dois estudos encontraram uma associação positiva e significativa entre o cronótipo vespertino e a DS ${ }^{16,57}$, com coeficientes beta de 0,349 e 0,231, respectivamente.

Um estudo concluiu que as pessoas identificadas como dependentes de smartphone apresentam piores hábitos alimentares que os grupos controle (coeficiente beta = $0,564 ; p<0,001)^{36}$. Além disso, três estudos encontraram uma associação positiva e significativa entre baixa atividade física/sedentarismo e DS17,36,38. Os coeficientes betas variaram entre 0,782 e 0,950. Um estudo sugere que os adolescentes que moram com os pais têm menos chances de serem dependentes de smartphone do que aqueles que moram sozinhos (beta $=-0,247 ; p<0,01$ ), o que pode estar relacionado ao monitoramento do uso do aparelho pelos pais ${ }^{57}$. Um estudo encontrou uma associação entre sexo desprotegido e DS em todas as faixas etárias, exceto em homens menores de 15 anos de idade (OR na mulher $=2,933, p<0,001$; OR no homem = 3,149, $p<0,001)^{53}$. Além disso, um estudo encontrou uma associação entre abuso de smartphone e baixo desempenho acadêmico (beta $=0,821 ; p<0,001)^{36}$.

Podemos inferir que os principais hábitos de vida associados à DS são cronótipo vespertino, baixa atividade física e sedentarismo. 
JULIA MACHADO KHOURY',2,3, JOÃO PEDRO SOUSA DRUMOND², LETÍCIA LOPES

DE CARVALHO E SILVA², MARIANE DA SILVA MELO ${ }^{1}$, ANANDA ARAUJO TEIXEIRA ${ }^{1,3,4}$, MAILA DE CASTRO LOURENC̣O DAS NEVES ${ }^{1,3,4}(1)$, FREDERICO DUARTE GARCIA ${ }^{1,3,4,5}$

1 Departamento de Saúde Mental, Faculdade de Medicina de Minas Gerais, Belo Horizonte, MG

2 Departamento de Clínica Médica, Faculdade de Saúde e Ecologia Humana, Vespasiano, MG.

3 Programa de Pós-Graduação em Medicina Molecular, Faculdade de Medicina, Universidade Federal de Minas Gerais (UFMG), Belo Horizonte, MG. ${ }^{4}$ INCT de Medicina Molecular, UFMG, Belo Horizonte, MG. ${ }^{5}$ Unité Inserm U1073, Rouen, França.

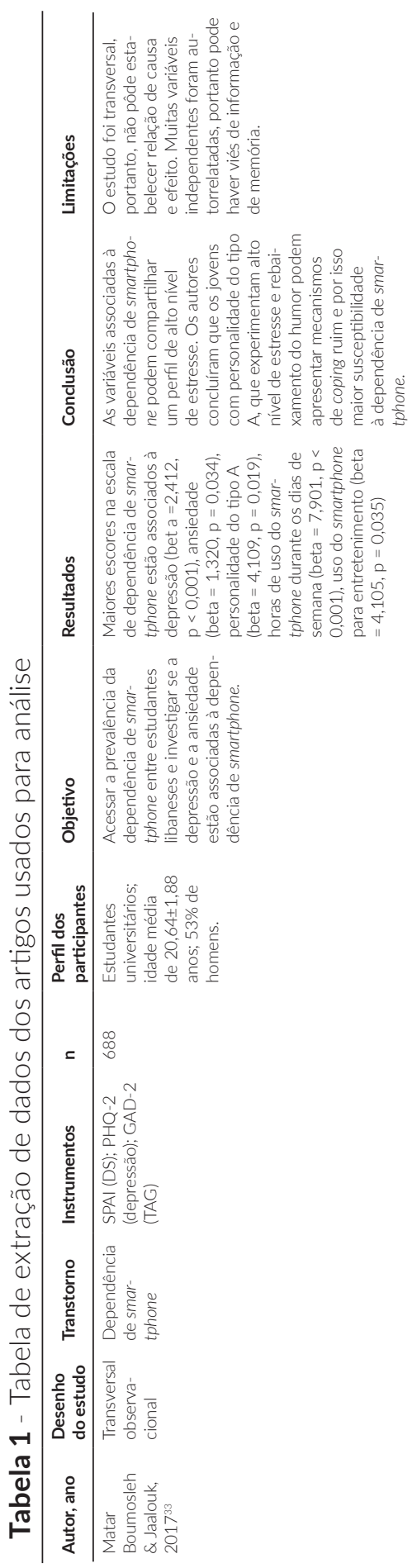

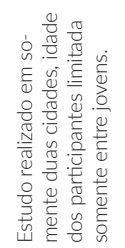
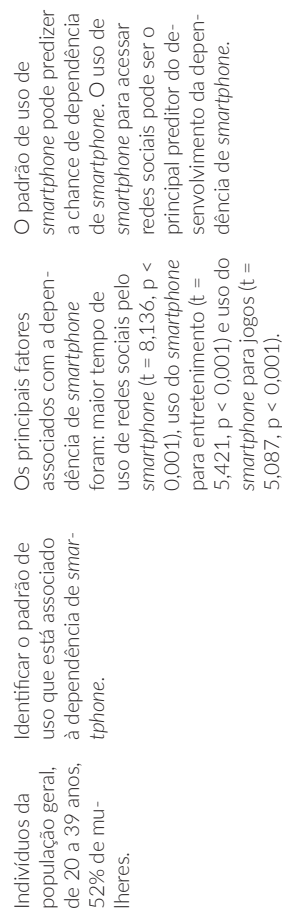

$\stackrel{\infty}{\sim}$

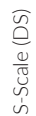

峁

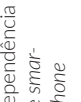

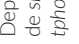

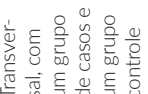

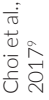
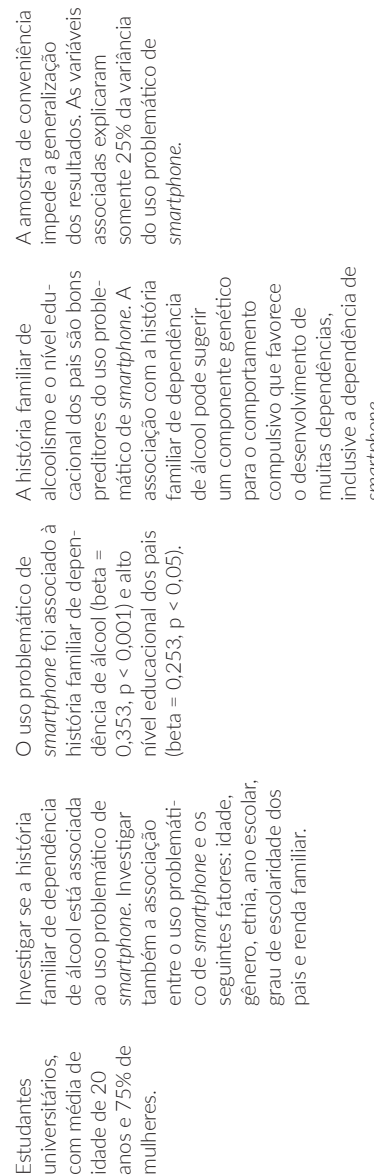

욱

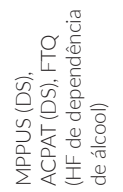

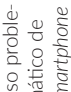

焉

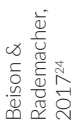

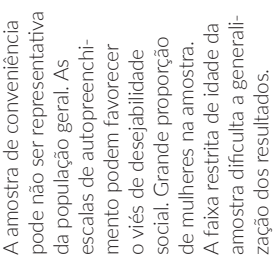

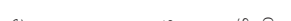

$\frac{8}{\pi}$

离

¿.

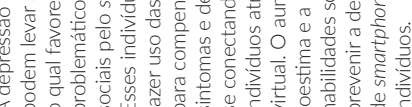

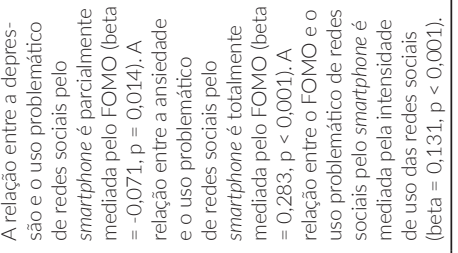

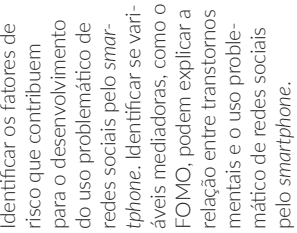

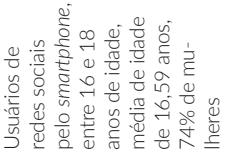

$\underset{\substack{\infty \\ \stackrel{\infty}{+}}}{\stackrel{\infty}{+}}$

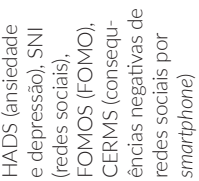

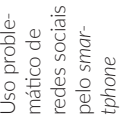

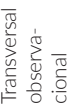

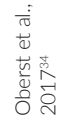




\section{ARTIGO DE REVISÃO}

JULIA MACHADO KHOURY JOÃO PEDRO SOUSA DRUMOND LETÍCIA LOPES DE CARVALHO E SILVA MARIANE DA SILVA MELO ANANDA ARAUJO TEIXEIRA MAILA DE CASTRO LOURENC̣O DAS NEVES FREDERICO DUARTE GARCIÁ
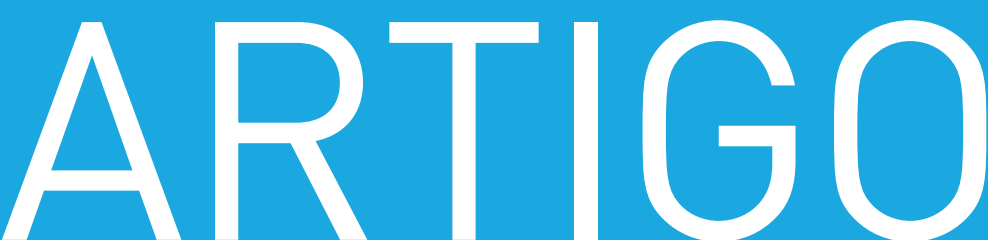

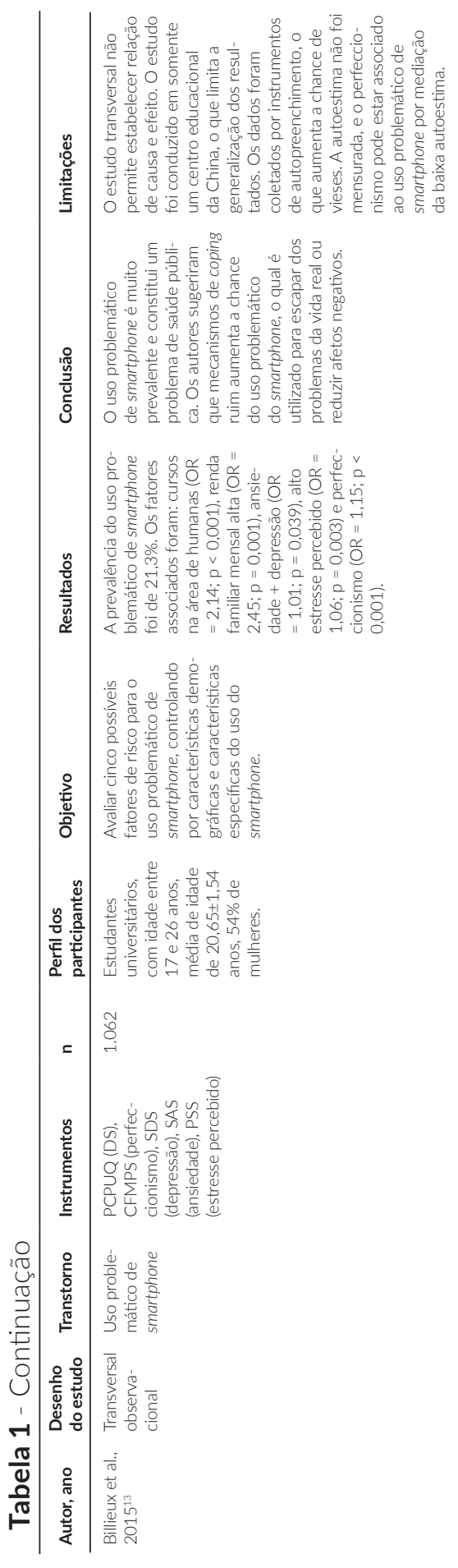

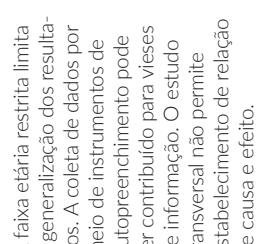

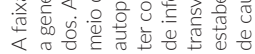

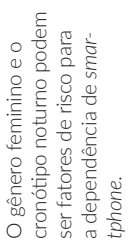

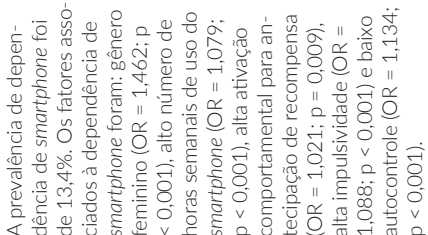

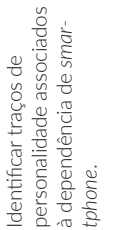

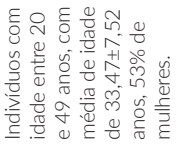

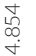

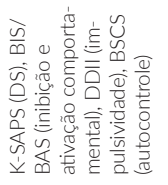

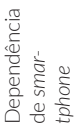

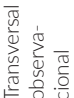

要

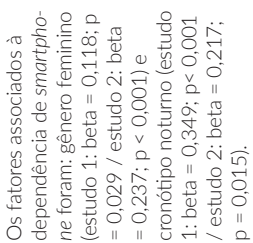

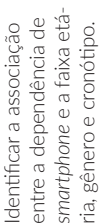

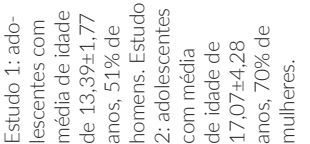

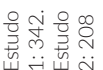

ษิ

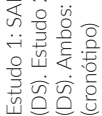

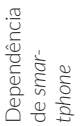

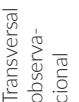

एँ

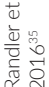

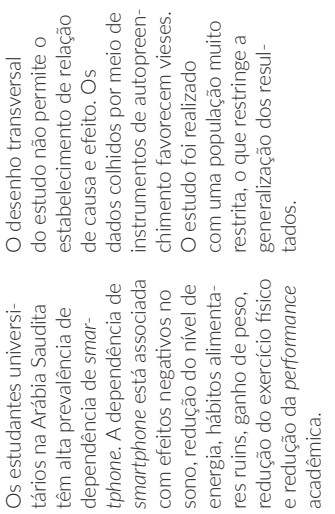

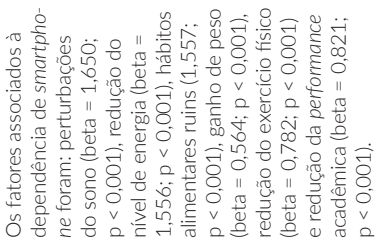

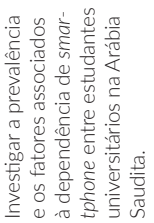

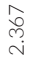

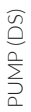

曹

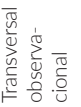

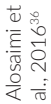


JULIA MACHADO KHOURY',2,3 (D, JOÃO PEDRO SOUSA DRUMOND², LETÍCIA LOPES

DE CARVALHO E SILVA², MARIANE DA SILVA MELO ${ }^{1}$, ANANDA ARAUJO TEIXEIRA1,3,4, MAILA DE CASTRO LOURENÇO DAS NEVES ${ }^{1,3,4}$ (D), FREDERICO DUARTE GARCIA ${ }^{1,3,4,5}$

${ }^{1}$ Departamento de Saúde Mental, Faculdade de Medicina de Minas Gerais, Belo Horizonte, MG.

2 Departamento de Clínica Médica, Faculdade de Saúde e Ecologia Humana, Vespasiano, MG.

3 Programa de Pós-Graduação em Medicina Molecular, Faculdade de Medicina, Universidade Federal de Minas Gerais (UFMG), Belo Horizonte, MG. ${ }^{4}$ INCT de Medicina Molecular, UFMG, Belo Horizonte, MG. ${ }^{5}$ Unité Inserm U1073, Rouen, França.

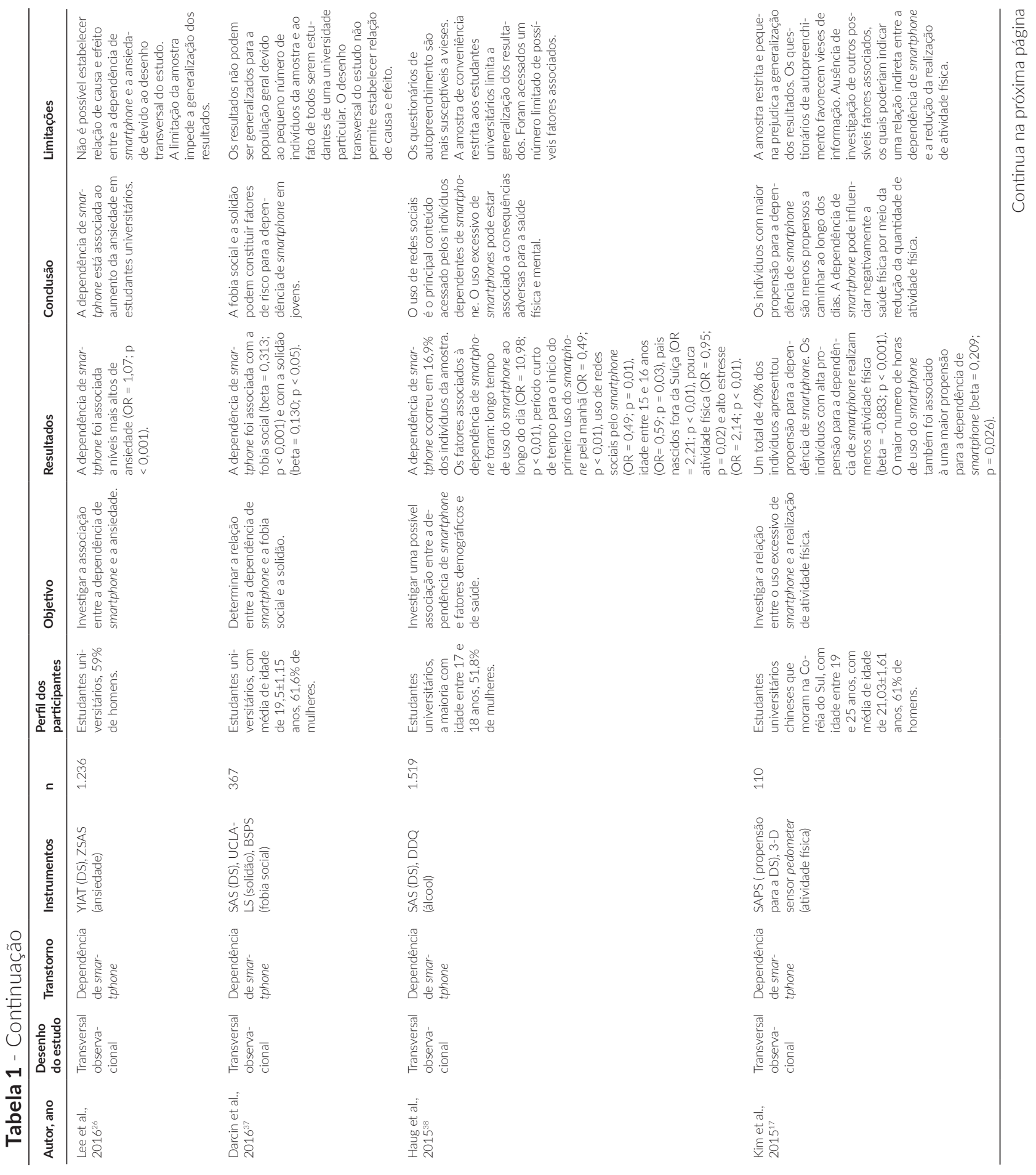




\section{ARTIGO DE REVISÃO}

JULIA MACHADO KHOURY JOÃO PEDRO SOUSA DRUMOND LETÍCIA LOPES DE CARVALHO E SILVA MARIANE DA SILVA MELO ANANDA ARAUJO TEIXEIRA MAILA DE CASTRO LOURENC̣O DAS NEVES FREDERICO DUARTE GARCIÁ
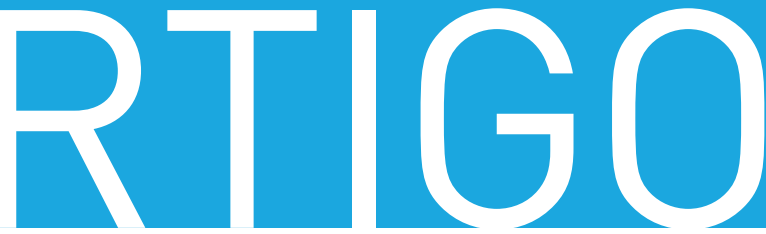

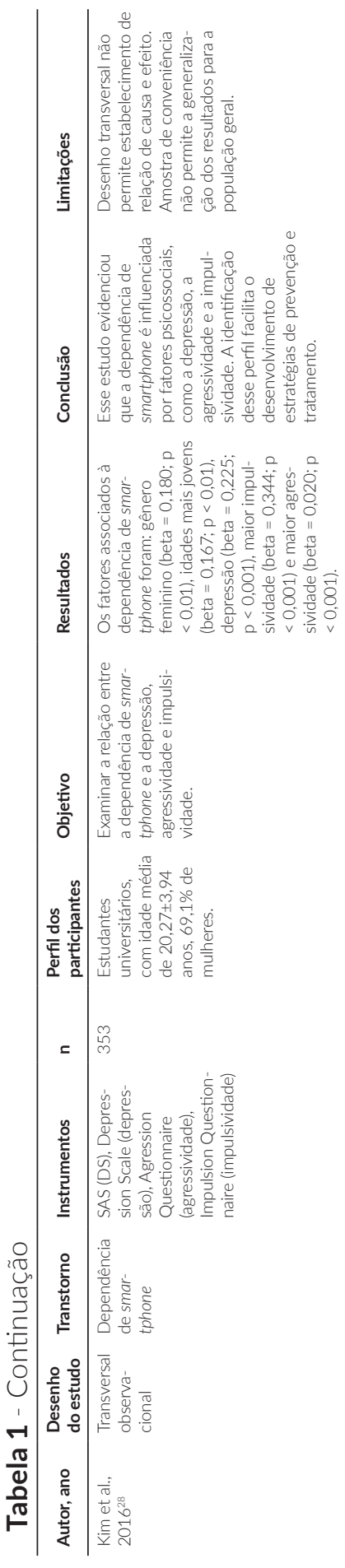

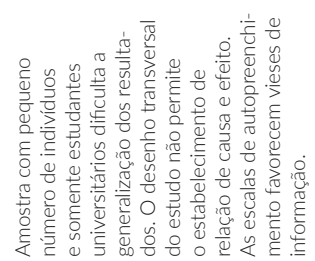

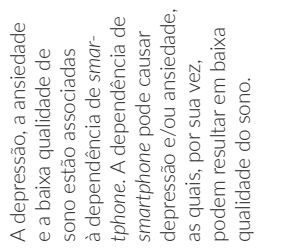

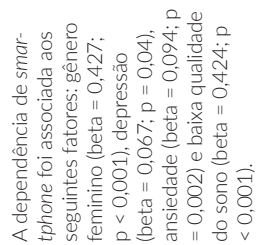

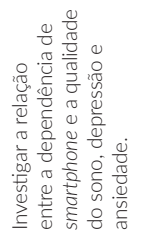

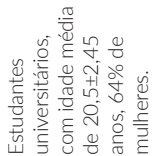

ले

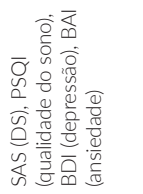

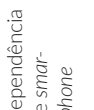

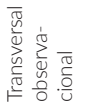

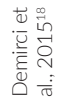
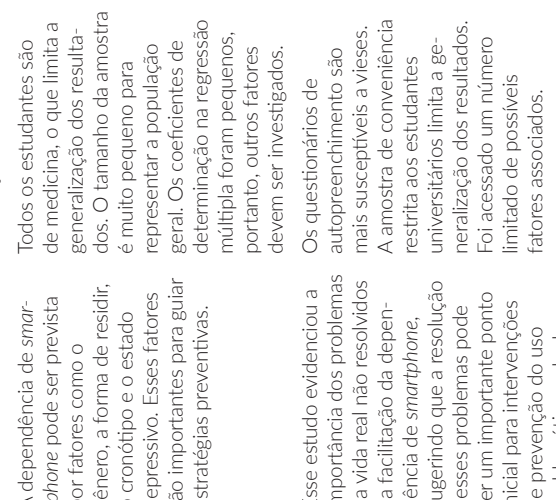

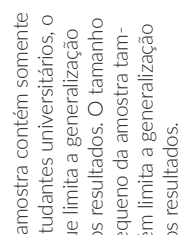

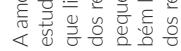
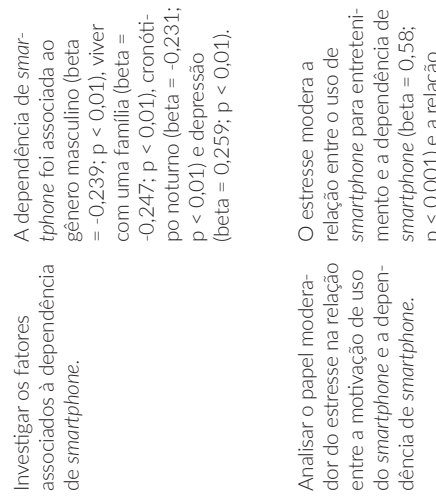

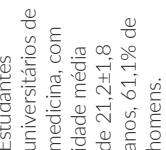

$\stackrel{\circ}{\circ}$

$\stackrel{\Upsilon}{\mathrm{W}} \overline{\mathrm{0}}$

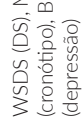

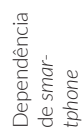

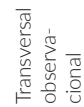

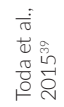

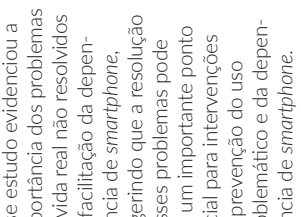

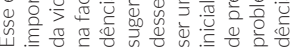

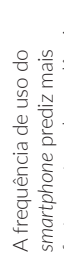

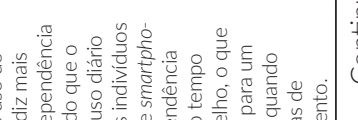

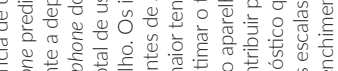
$\begin{array}{llll} & \\ 0 & \\ 0\end{array}$

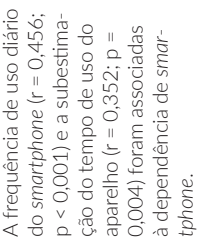

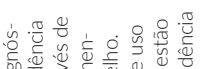

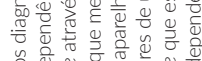
은

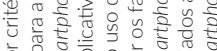

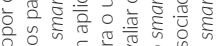

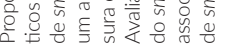

\begin{tabular}{|c|c|}
\hline 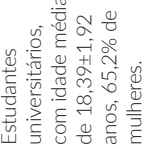 & 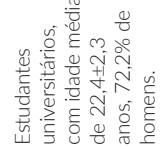 \\
\hline
\end{tabular}

$\stackrel{9}{\dot{v}}$

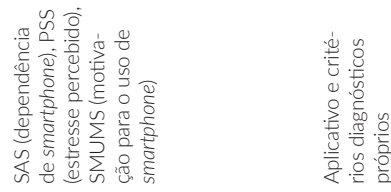

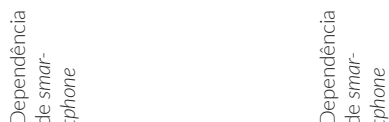

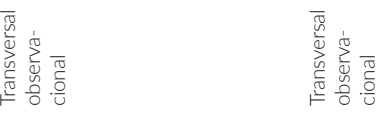

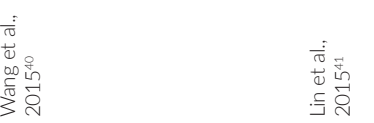


JULIA MACHADO KHOURY1,2,3, JOÃO PEDRO SOUSA DRUMOND², LETÍCIA LOPES

DE CARVALHO E SILVA ${ }^{2}$, MARIANE DA SILVA MELO ${ }^{1}$, ANANDA ARAUJO TEIXEIRA ${ }^{1,3,4}$, MAILA DE CASTRO LOURENC̣O DAS NEVES ${ }^{1,3,4}\left[\right.$, FREDERICO DUARTE GARCIA ${ }^{1,3,4,5}$

1 Departamento de Saúde Mental, Faculdade de Medicina de Minas Gerais, Belo Horizonte, MG

2 Departamento de Clínica Médica, Faculdade de Saúde e Ecologia Humana, Vespasiano, MG.

3 Programa de Pós-Graduação em Medicina Molecular, Faculdade de Medicina, Universidade Federal de Minas Gerais (UFMG), Belo Horizonte, MG. ${ }^{4}$ INCT de Medicina Molecular, UFMG, Belo Horizonte, MG. ${ }^{5}$ Unité Inserm U1073, Rouen, França.

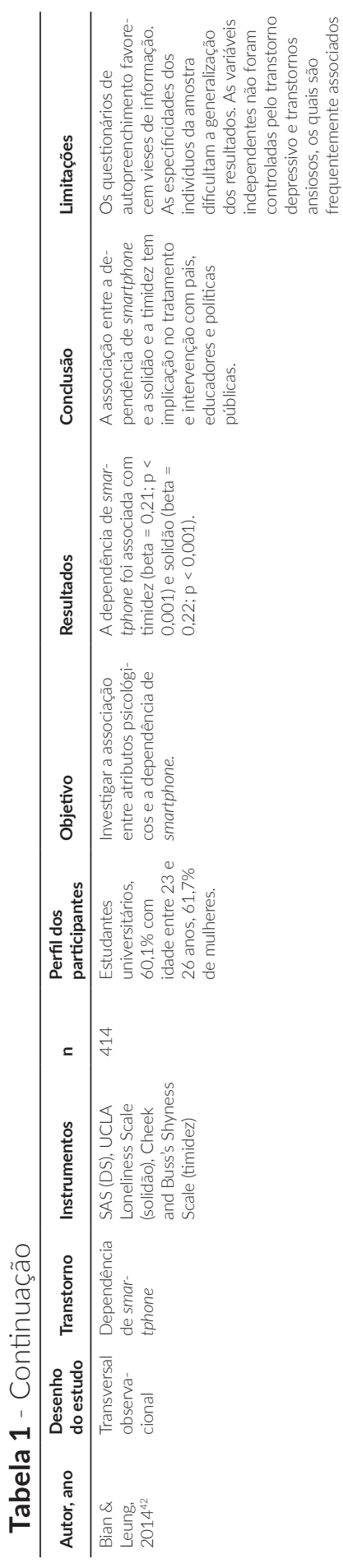

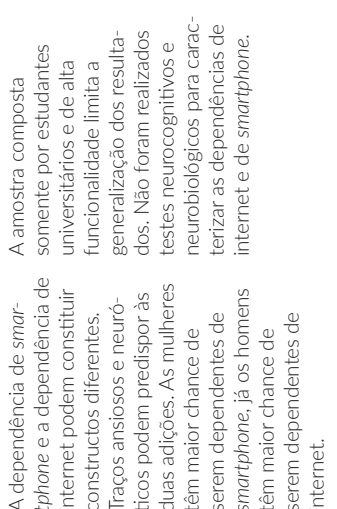

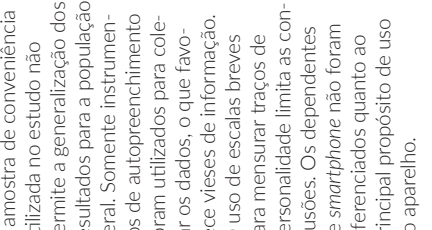

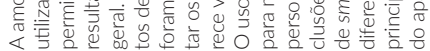
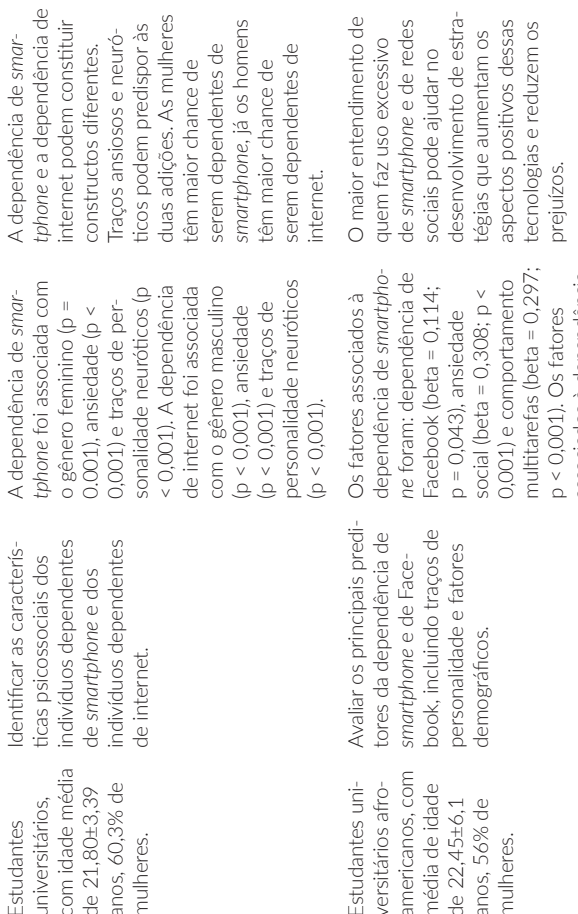

$\stackrel{\infty}{\forall}$
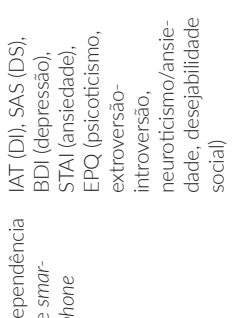

要

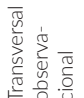

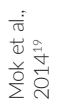
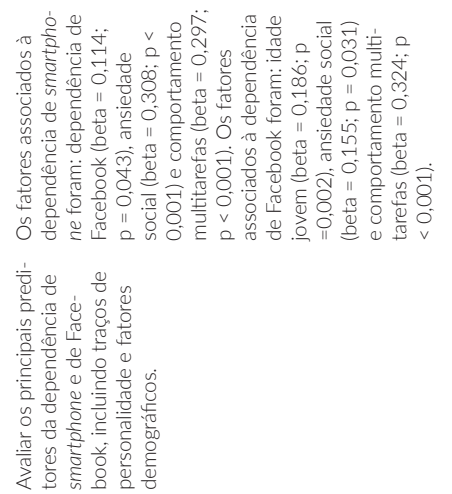

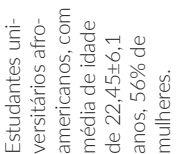

产

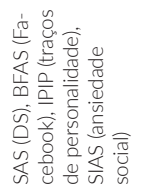

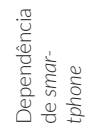

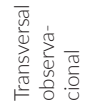

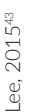

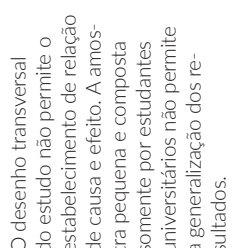

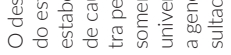

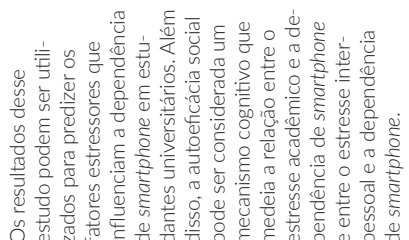

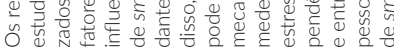
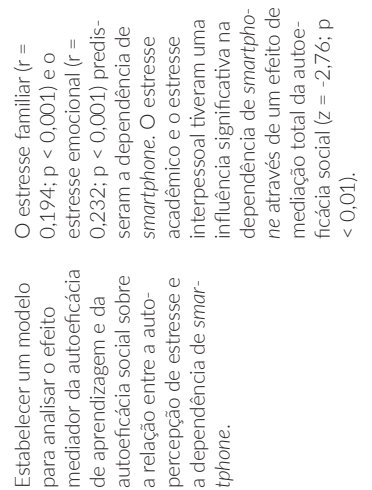

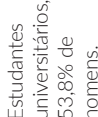

$\stackrel{\infty}{\infty}$

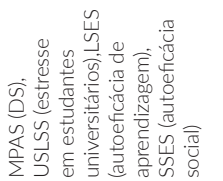

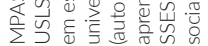

曹 


\section{ARTIGO DE REVISÃO}

JULIA MACHADO KHOURY JOÃO PEDRO SOUSA DRUMOND LETÍCIA LOPES DE CARVALHO E SILVA MARIANE DA SILVA MELO ANANDA ARAUJO TEIXEIRA MAILA DE CASTRO LOURENCCO DAS NEVES FREDERICO DUARTE GARCIÁ
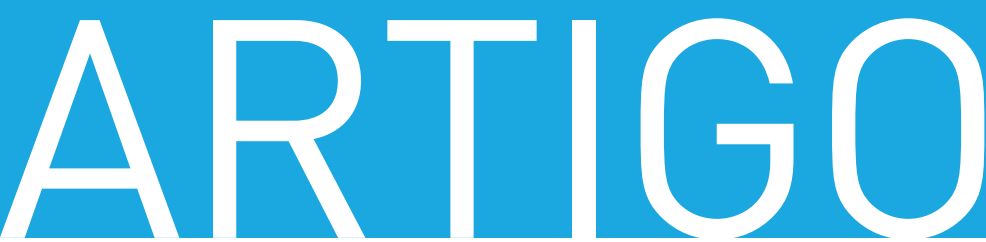

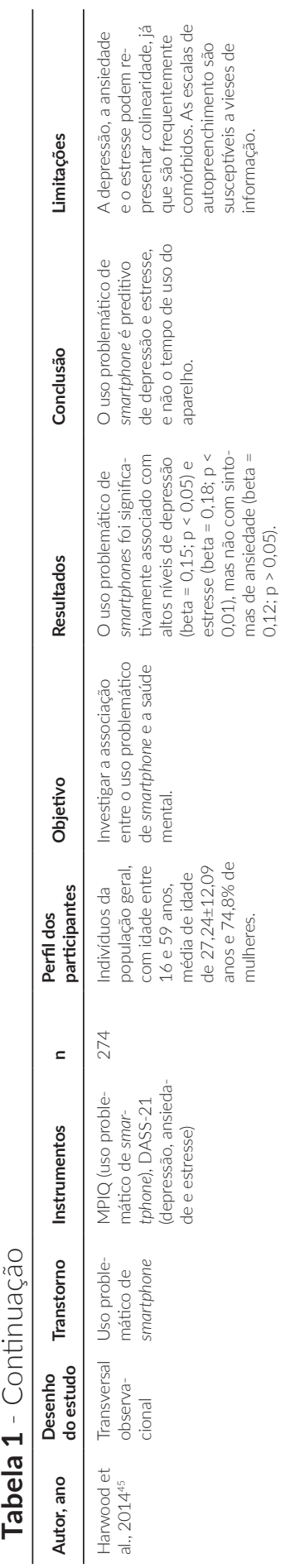

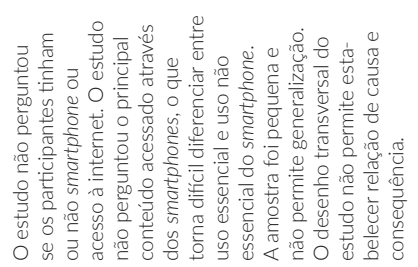
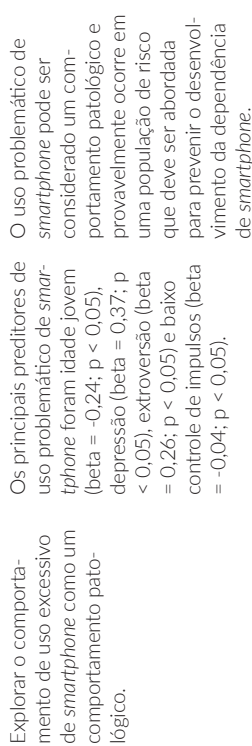

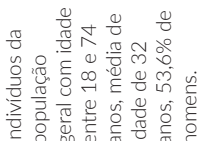

ळু

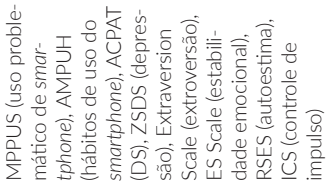

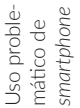

焉

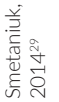

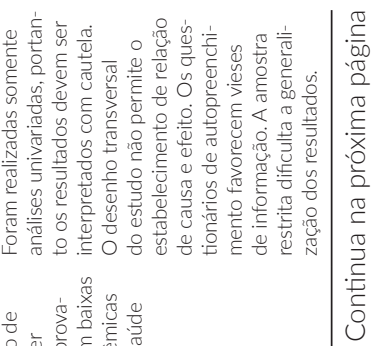

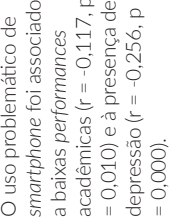

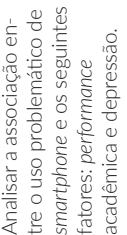

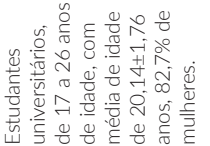

ஓ

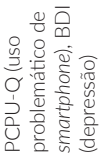

응 음

号
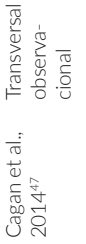
JULIA MACHADO KHOURY1,2,3

DE CARVALHO E SILVA ${ }^{2}$, MARIANE DA SILVA MELO ${ }^{1}$, ANANDA ARAUJO TEIXEIRA ${ }^{1,3,4}$, MAILA DE CASTRO LOURENC̣O DAS NEVES ${ }^{1,3,4}\left[\right.$, FREDERICO DUARTE GARCIA ${ }^{1,3,4,5}$

1 Departamento de Saúde Mental, Faculdade de Medicina de Minas Gerais, Belo Horizonte, MG

2 Departamento de Clínica Médica, Faculdade de Saúde e Ecologia Humana, Vespasiano, MG.

3 Programa de Pós-Graduação em Medicina Molecular, Faculdade de Medicina, Universidade Federal de Minas Gerais (UFMG), Belo Horizonte, MG. ${ }^{4}$ INCT de Medicina Molecular, UFMG, Belo Horizonte, MG. ${ }^{5}$ Unité Inserm U1073, Rouen, França.
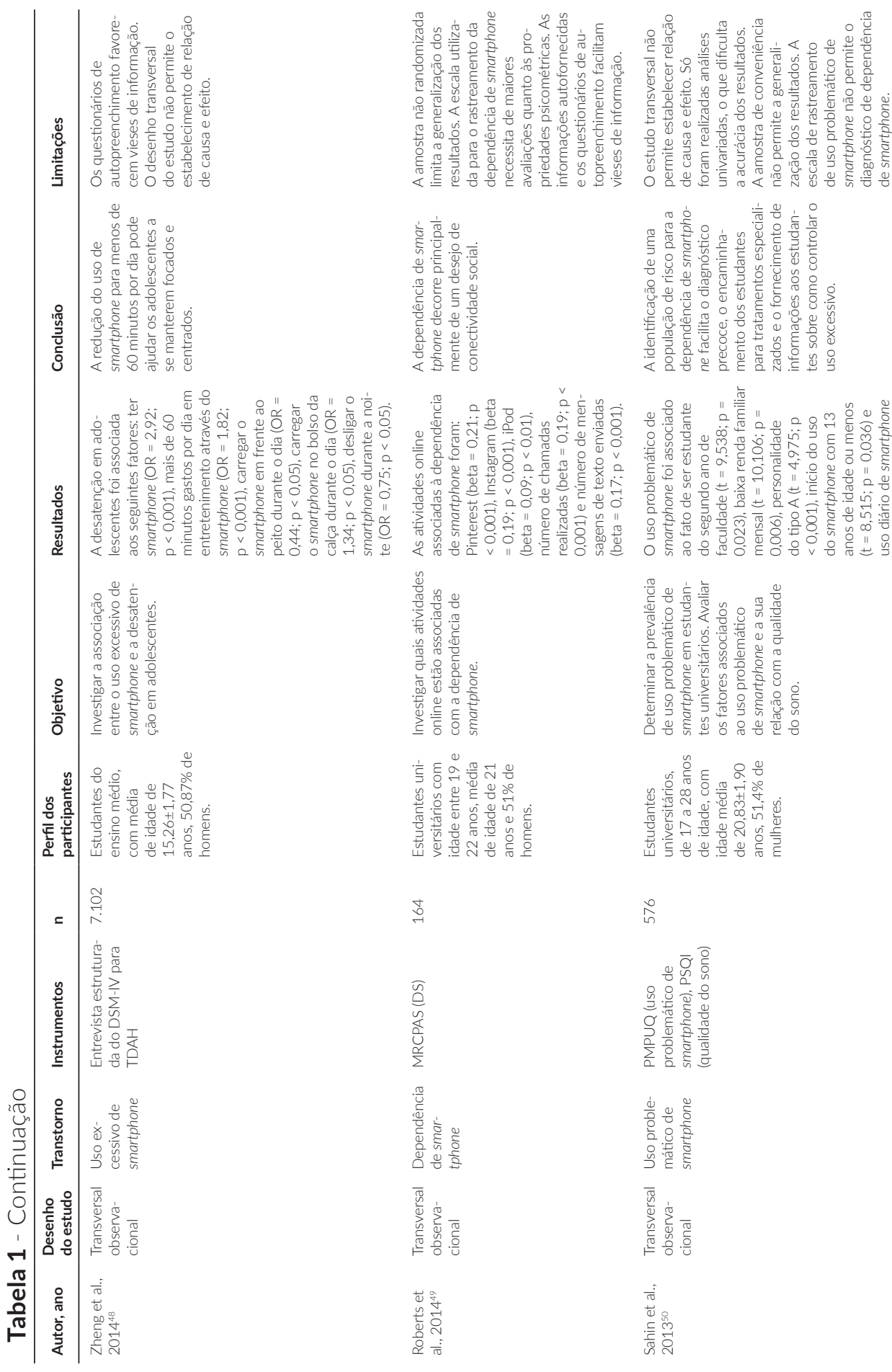

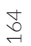

$i$

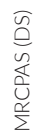

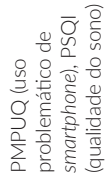

营

这密高

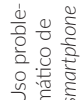

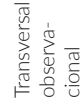

$\overline{\mathrm{W}}$

竞离产

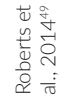

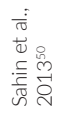




\section{ARTIGO DE REVISÃO}

JULIA MACHADO KHOURY JOÃO PEDRO SOUSA DRUMOND LETÍCIA LOPES DE CARVALHO E SILVA MARIANE DA SILVA MELO ANANDA ARAUJO TEIXEIRA MAILA DE CASTRO LOURENC̣O DAS NEVES FREDERICO DUARTE GARCIÁ
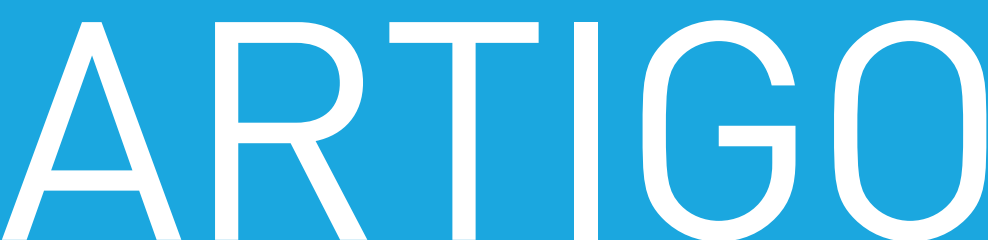

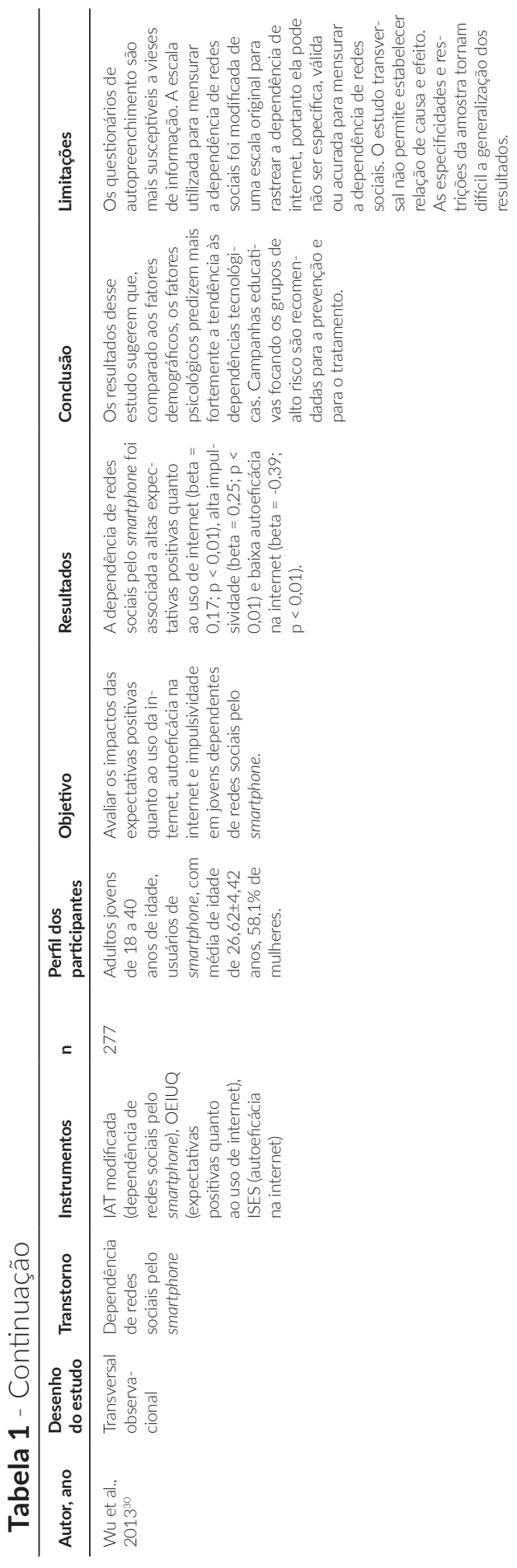

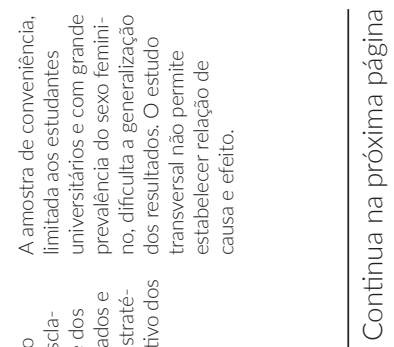

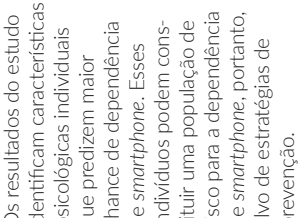

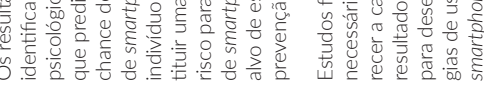
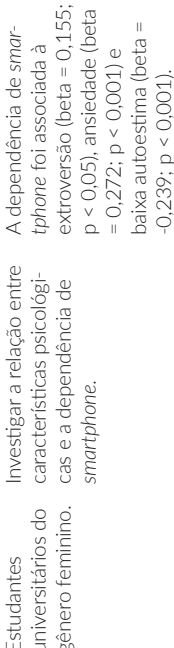

๙े
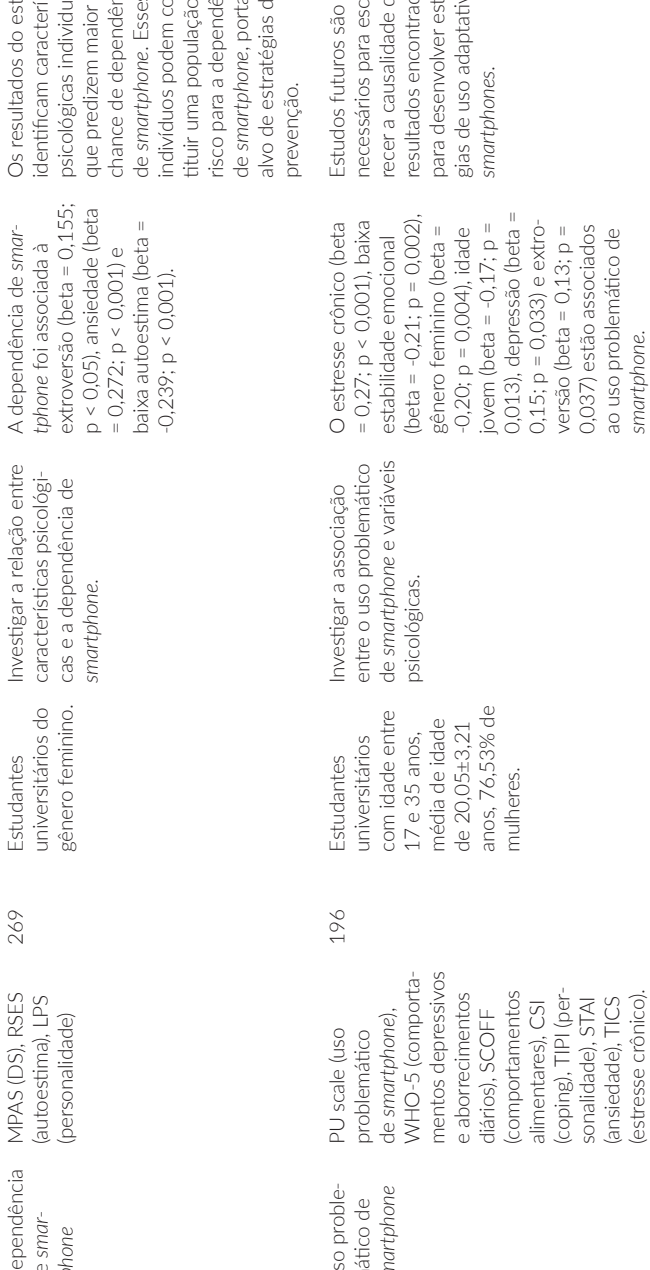

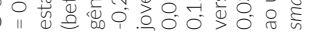
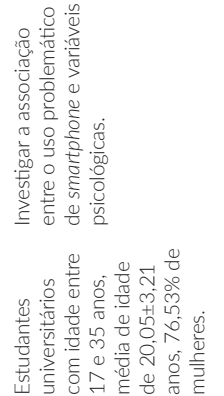

$\stackrel{\circ}{\frac{2}{-1}}$

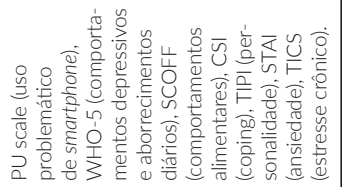

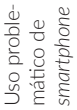

焉离离

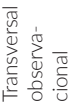

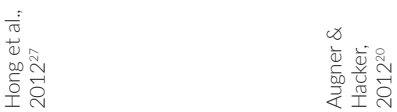


JULIA MACHADO KHOURY',2,3 (D, JOÃO PEDRO SOUSA DRUMOND², LETÍCIA LOPES

DE CARVALHO E SILVA ${ }^{2}$, MARIANE DA SILVA MELO ${ }^{1}$, ANANDA ARAUJO TEIXEIRA ${ }^{1,3,4}$, MAILA DE CASTRO LOURENÇO DAS NEVES ${ }^{1,3,4}$, FREDERICO DUARTE GARCIA ${ }^{1,3,4,5}$

${ }^{1}$ Departamento de Saúde Mental, Faculdade de Medicina de Minas Gerais, Belo Horizonte, MG. 2 Departamento de Clínica Médica, Faculdade de Saúde e Ecologia Humana, Vespasiano, MG. 3 Programa de Pós-Graduação em Medicina Molecular, Faculdade de Medicina, Universidade Federal de Minas Gerais (UFMG), Belo Horizonte, MG. ${ }^{4}$ INCT de Medicina Molecular, UFMG, Belo Horizonte, MG. ${ }^{5}$ Unité Inserm U1073, Rouen, França.

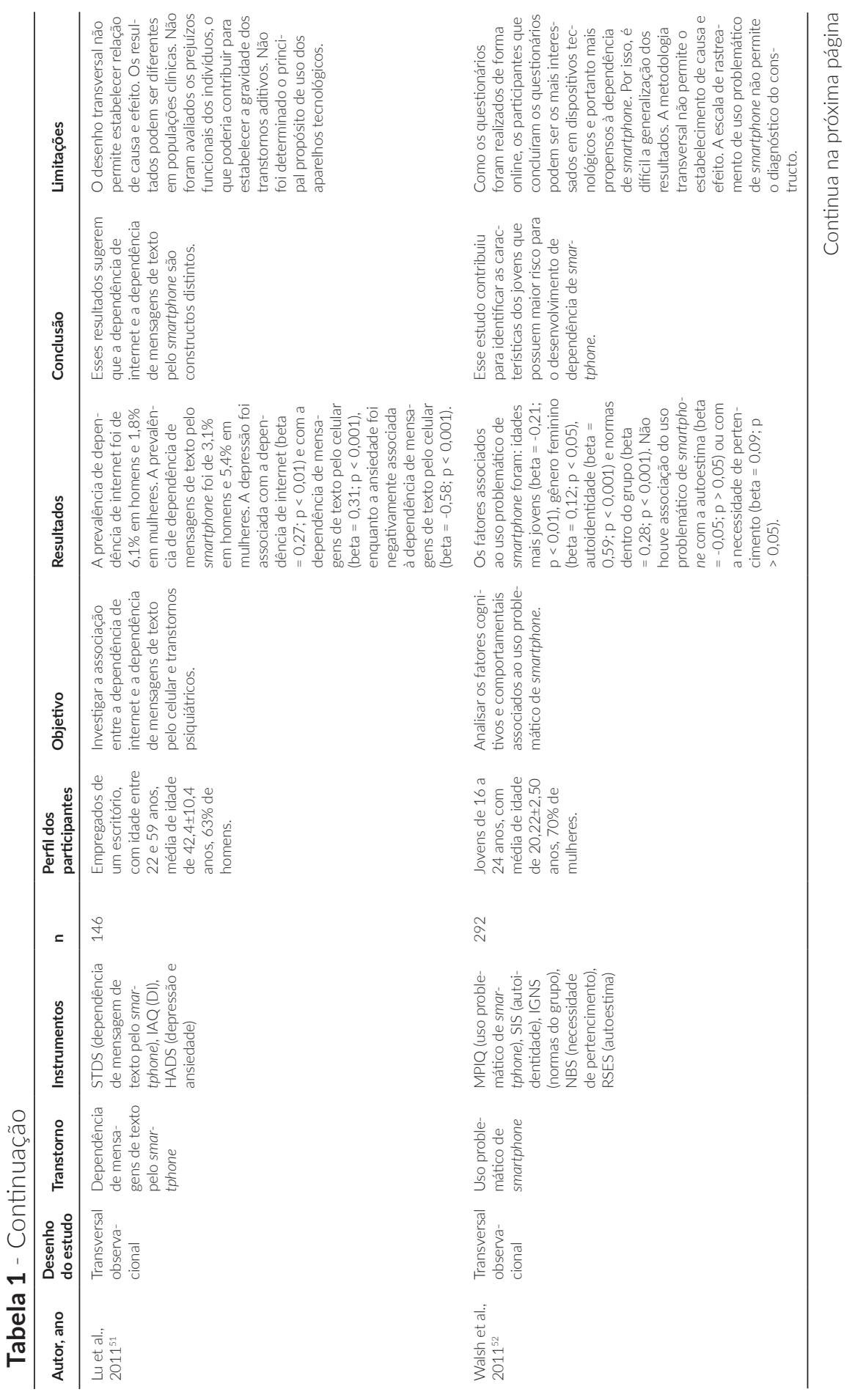




\section{ARTIGO DE REVISÃO}

\section{JULIA MACHADO KHOURY} JOÃO PEDRO SOUSA DRUMOND LETÍCIA LOPES DE CARVALHO E SILVA MARIANE DA SILVA MELO ANANDA ARAUJO TEIXEIRA MAILA DE CASTRO LOURENÇO DAS NEVES FREDERICO DUARTE GARCIA
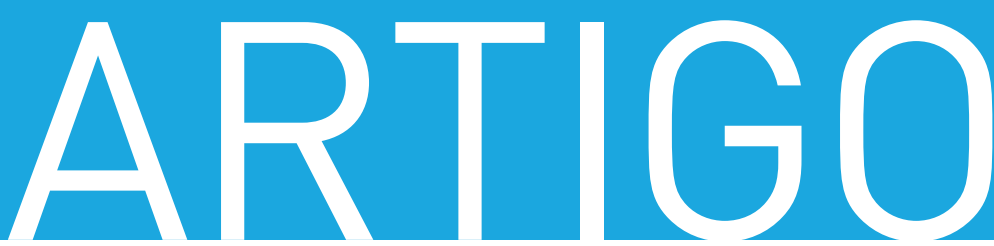
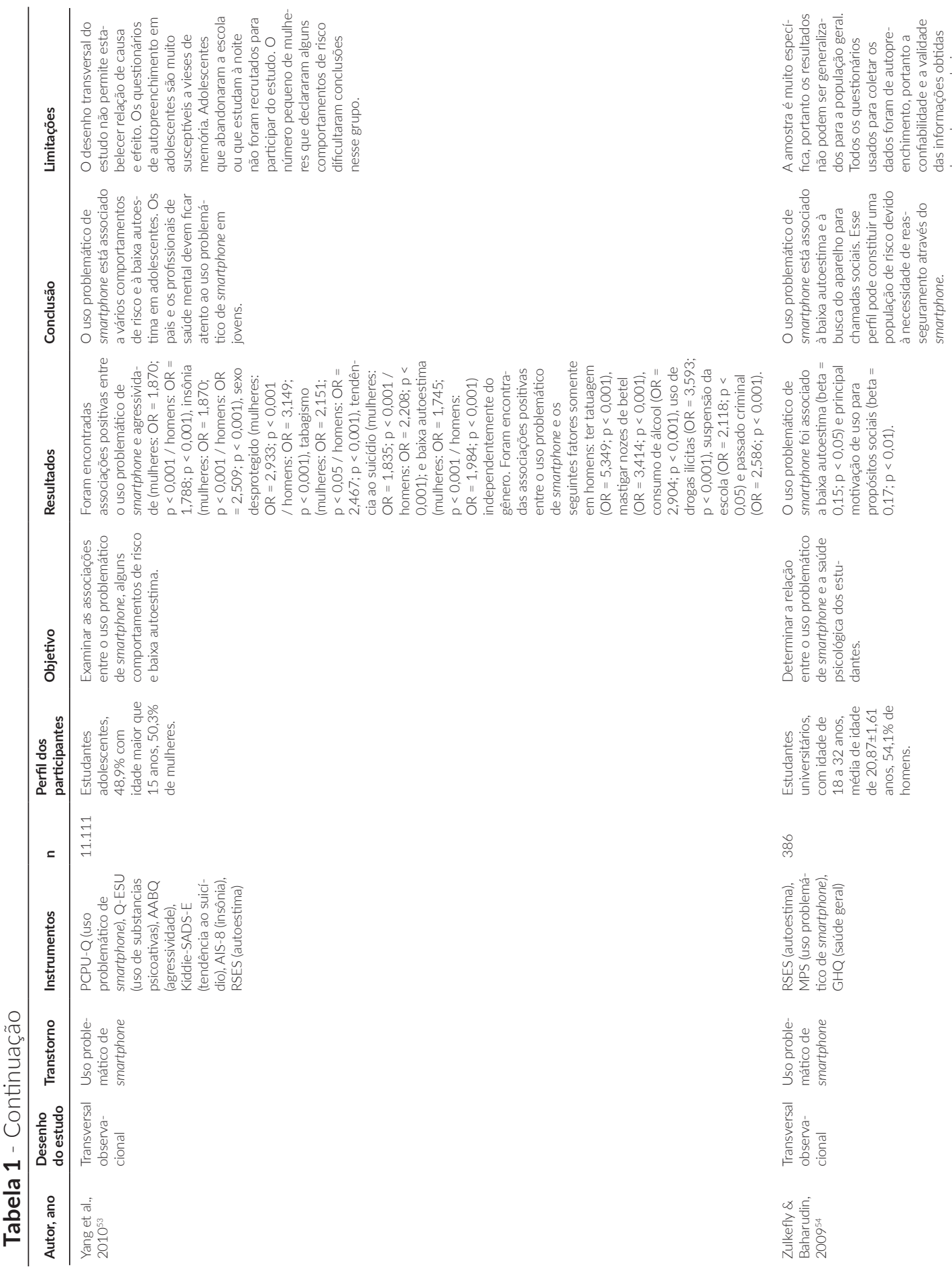

品

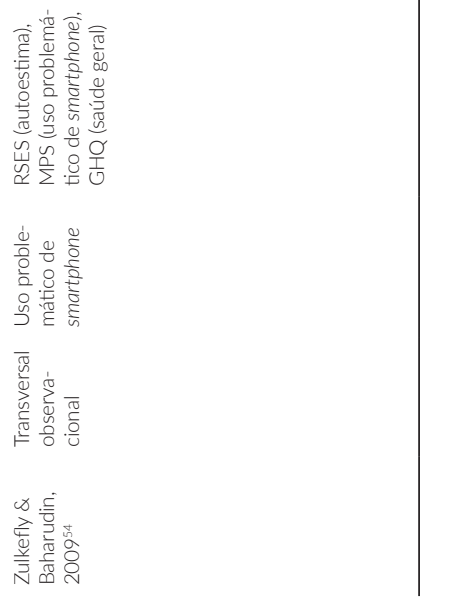


JULIA MACHADO KHOURY',2,3 (D, JOÃO PEDRO SOUSA DRUMOND², LETÍCIA LOPES

DE CARVALHO E SILVA², MARIANE DA SILVA MELO ${ }^{1}$, ANANDA ARAUJO TEIXEIRA1,3,4,

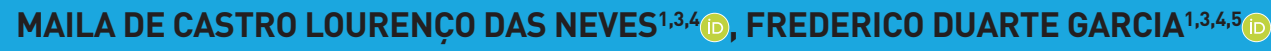

${ }^{1}$ Departamento de Saúde Mental, Faculdade de Medicina de Minas Gerais, Belo Horizonte, MG. 2 Departamento de Clínica Médica, Faculdade de Saúde e Ecologia Humana, Vespasiano, MG. 3 Programa de Pós-Graduação em Medicina Molecular, Faculdade de Medicina, Universidade Federal de Minas Gerais (UFMG), Belo Horizonte, MG. ${ }^{4}$ INCT de Medicina Molecular, UFMG, Belo Horizonte, MG. ${ }^{5}$ Unité Inserm U1073, Rouen, França.

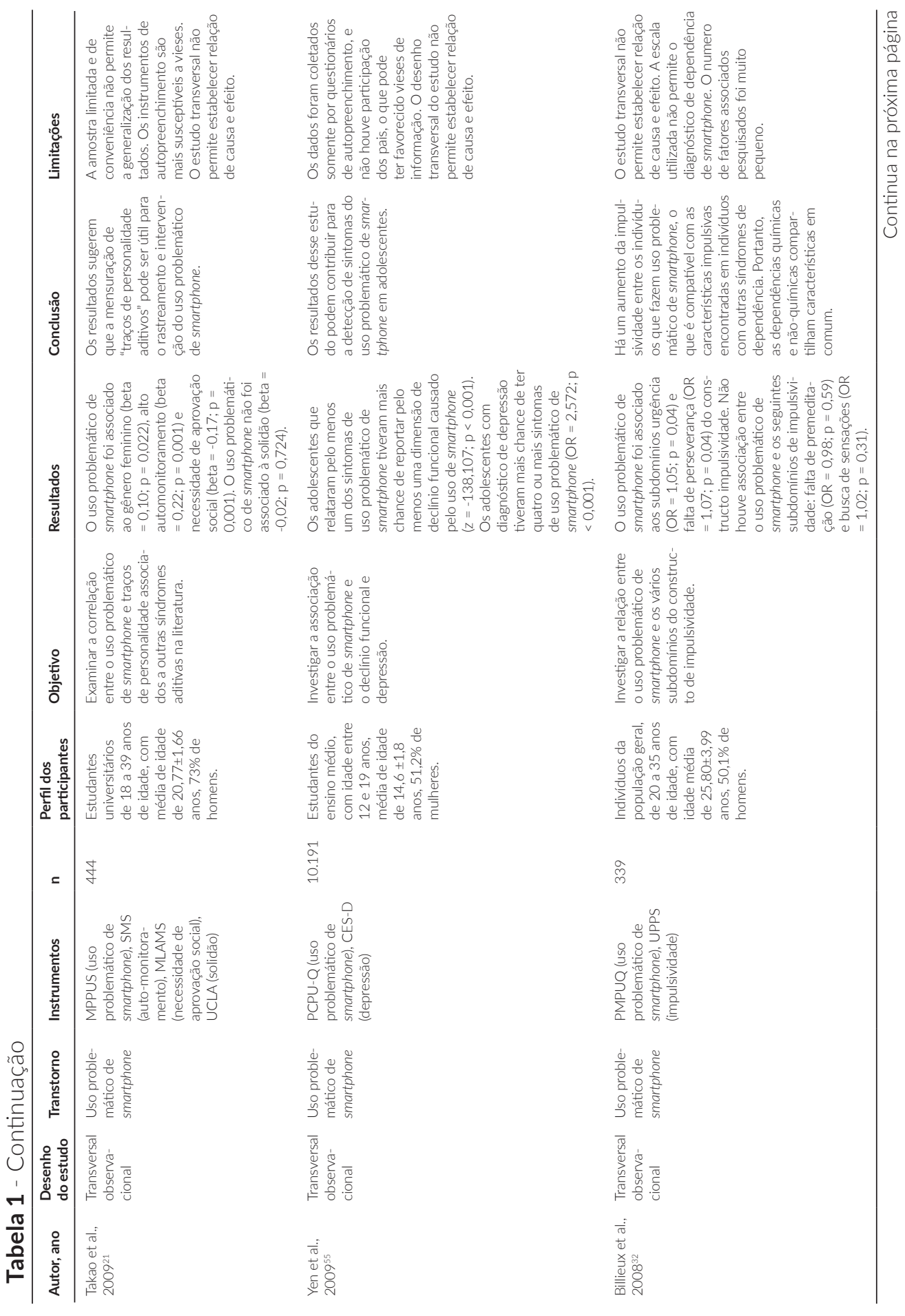




\section{ARTIGO DE REVISÃO}

JULIA MACHADO KHOURY JOÃO PEDRO SOUSA DRUMOND LETÍCIA LOPES DE CARVALHO E SILVA MARIANE DA SILVA MELO ANANDA ARAUJO TEIXEIRA MAILA DE CASTRO LOURENC̣O DAS NEVES FREDERICO DUARTE GARCIÁ
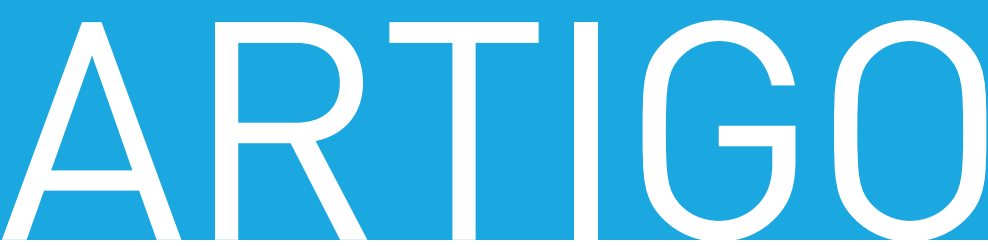

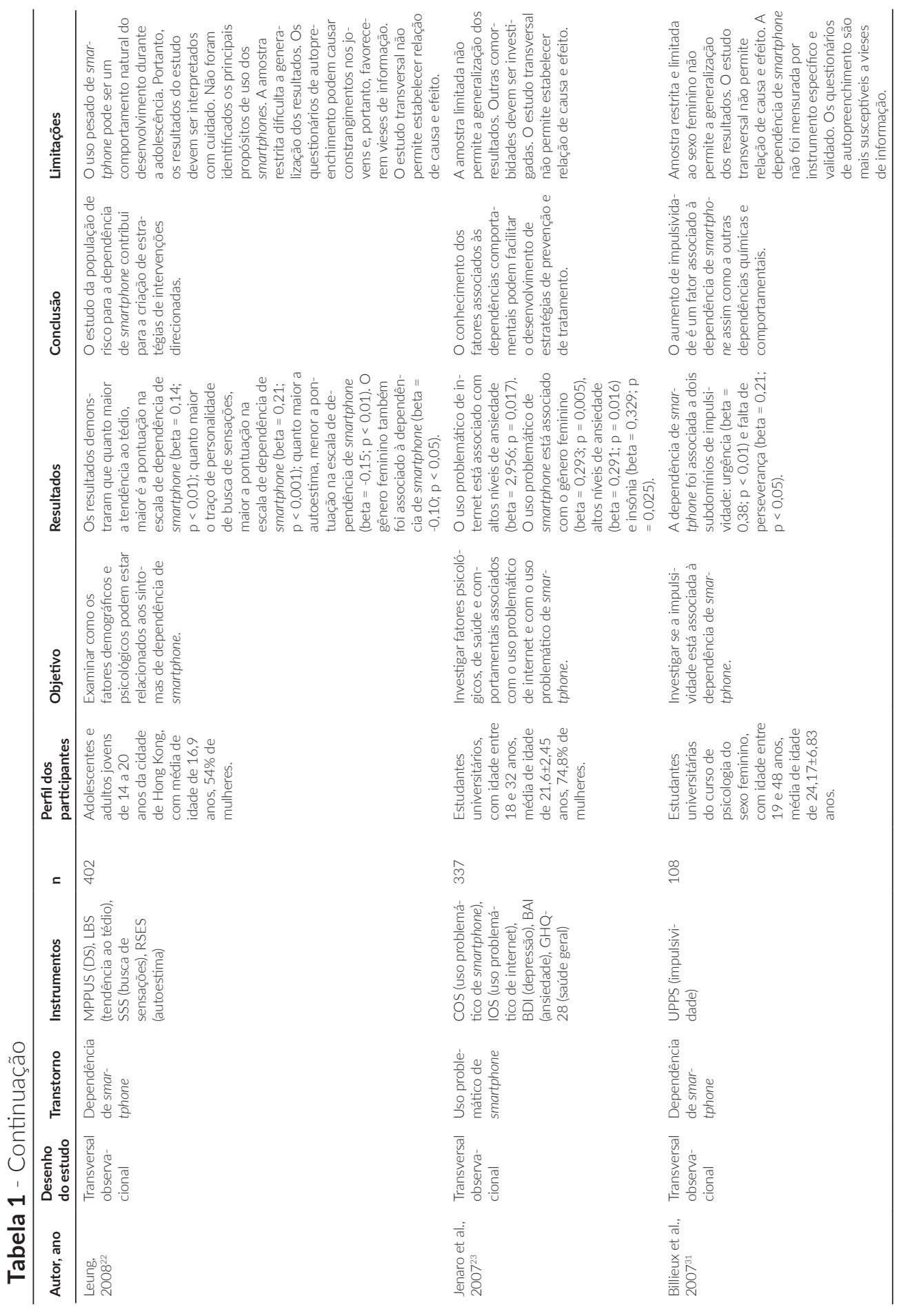

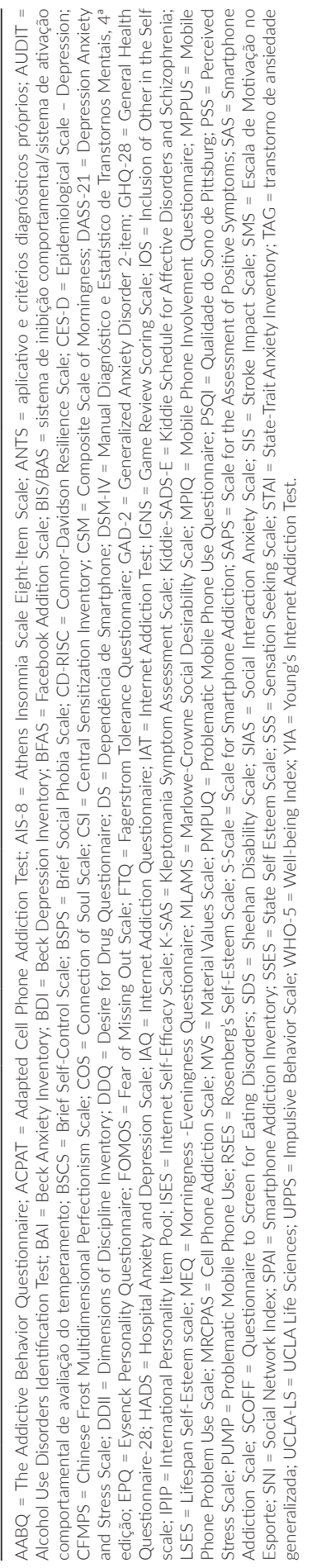




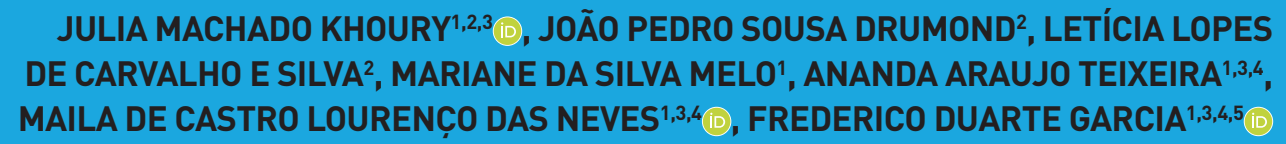

Departamento de Saúde Mental, Faculdade de Medicina de Minas Gerais, Belo Horizonte, MG Departamento de Clínica Médica, Faculdade de Saúde e Ecologia Humana, Vespasiano, MG. Programa de Pós-Graduação em Medicina Molecular, Faculdade de Medicina, Universidade Federal de Minas Gerais (UFMG), Belo Horizonte, MG. ${ }^{4}$ INCT de Medicina Molecular, UFMG, Belo Horizonte, MG. ${ }^{5}$ Unité Inserm U1073, Rouen, França.

Hábitos relacionados ao uso de smartphone

Seis estudos encontraram uma associação positiva e significativa entre o uso de smartphone por um longo período de tempo ao longo do dia (mais de 4 horas por dia) e a DS17,28,33,38,41,50. Em 2014, Zheng et al. ${ }^{48}$ desenvolveram um estudo com adolescentes na China, no qual a desatenção estava associada àqueles que gastam mais de 60 minutos por dia utilizando smartphones $(\mathrm{OR}=1,82 ; \mathrm{p}$ $<0,001)$. Além disso, Lin et al. reportaram que as pessoas que subestimam o seu tempo de uso de smartphone têm um risco aumentado de apresentar DS $(r=0,352$; $p=0,004)^{41}$

Em 2015, Haug et al. ${ }^{38}$ desenvolveram um estudo com adolescentes e adultos jovens na Suiça, a fim de avaliar os indicadores de uso de smartphone associados à DS. Os autores encontraram uma correlação negativa entre o tempo até o primeiro uso do smartphone pela manhã e o risco de ser identificado com portador de DS. O maior risco foi encontrado em indivíduos que apresentam um intervalo inferior a 10 minutos $(O R=0,49 ; p<0,01)$.

Zheng etal. ${ }^{48}$ associaram algumas posições de transporte do smartphone à DS. Os autores concluíram que carregar o smartphone em frente ao tórax ou no bolso da calça era um hábito significativamente associado à $D S(O R=0,44$; $p<0,05$ e $\mathrm{OR}=1,34 ; \mathrm{p}<0,05$, respectivamente). Um estudo associou a idade do primeiro uso de smartphone à DS. Sahin et al..$^{50}$ conduziram um estudo com estudantes da Universidade Sakarya e aferiram que indivíduos que ganharam o primeiro smartphone antes dos 13 anos de idade têm maior chance de serem dependentes de smartphone do que aqueles cuja idade de uso pela primeira vez foi de 16 anos ou mais ( $t=8,515 ; p=0,03$ ).

Assim, alguns hábitos relacionados ao uso de smartphone podem estar associados a uma maior chance de DS. O uso diário do smartphone por mais de 4 horas é um dos principais fatores associados à DS. Outros hábitos, como carregar o smartphone na frente do peito ou nos bolsos das calças, o primeiro contato com o smartphone antes de 13 anos de idade e o período de tempo até o primeiro uso pela manhã inferior a 10 minutos também foram associados à DS.

\section{Conteúdo acessado pelo smartphone}

Diferente da internet, que depende de uma busca ativa, os aplicativos permitem uma interação direta entre os algoritmos e provêm acesso aos diversos acessíveis pelo smartphone. Alguns desses conteúdos podem ser manipulados ou podem se associar mais facilmente à DS. Cinco estudos sugeriram que os principais conteúdos utilizados pelos dependentes de smartphone são as redes sociais ${ }^{10,35,49,54}$. Um estudo encontrou uma correlação entre o número de mensagens de texto enviadas por dia e o risco de DS (beta $=0,17 ; p<0,001$ ). Em 2014, Roberts et al. ${ }^{49}$ associaram o uso abusivo das seguintes redes sociais e utilizações do smartphone com a DS: Pinterest (beta $=0,21 ; p<0,001$ ), Instagram (beta $=0,19 ; p<0,001$ ), iPod (beta $=0,29 ; p<0,01$ ), número de chamadas (beta = 0,1; $p<0,001$ ) e número de mensagens de texto (beta $=0,1 ; p<0,001)$. Um estudo encontrou uma associação positiva entre a utilização de jogos eletrônicos por meio de smartphones e a DS $(t=5,087 ; p<0,001)^{9}$.

Portanto, podemos concluir que os principais conteúdos associados à DS são as redes sociais.

\section{Propósito de uso}

O uso do smartphone para fins de conexão social foi associado à DS em cinco estudos 9,35,38,49,54. Em 2009, Zukefly \& Baharudin ${ }^{54}$ sugeriram que os estudantes cuja principal motivação para o uso do smartphone é para fins sociais têm maiores chances de serem dependentes de smartphone (beta $=0,17 ; p<0,01$ ).

Cinco estudos sugeriram que a DS está associada ao uso de smartphone para fins de entretenimento 9,10,33,40,48. O estudo realizado por Wang et al. ${ }^{40}$ sugeriram que o nível de estresse modera a relação entre o uso de smartphone para fins de entretenimento e a DS (beta = 0,58; $p<0,001)$. Além disso, o estudo de Zheng et al. ${ }^{48}$ evidenciou que o uso de smartphones por mais de 60 minutos por dia com o propósito de entretenimento está associado à DS $(O R=1,82 ; p<0,001)$. Em 2017, Matar Boumosleh \& Jaalouk ${ }^{33}$ sugeriram que a finalidade de entretenimento estava associada à DS em estudantes universitários (beta $=4,105, p=0,035$ ). Além disso, Choi et al. ${ }^{9}$ encontraram dois propósitos de uso de smartphones associados à DS: entretenimento ( $t=5,421$, $p<0,001)$ e jogos ( $t=5,087, p<0,001)$. Em 2015, Wang et al. ${ }^{40}$ concluíram que indivíduos estressados, cujo entretenimento era o propósito do uso de smartphones, eram mais propensos a desenvolver DS (beta $=0,58$; $p<0,001)$. Finalmente, Zheng et al. ${ }^{48}$ associaram o déficit 


\section{ARTIGO DE REVISÃO}

\section{JULIA MACHADO KHOURY \\ JOÃO PEDRO SOUSA DRUMOND \\ LETÍCIA LOPES DE CARVALHO E SILVA \\ MARIANE DA SILVA MELO \\ ANANDA ARAUJO TEIXEIRA \\ MAILA DE CASTRO LOURENC̣O DAS NEVES \\ FREDERICO DUARTE GARCIA}
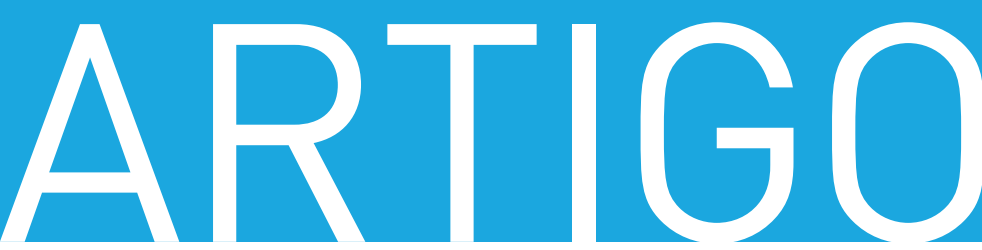

de atenção ao tempo gasto em jogos e entretenimento através dos smartphones (mais de 60 minutos por dia) $(\mathrm{OR}=1,82 ; \mathrm{p}<0,001)$.

O uso de smartphones com o objetivo de escapar da vida real foi outro fator associado positivamente à DS em estudantes universitários chineses (beta $=0,42$; $\mathrm{p}<0,001)^{40}$.

Portanto, o uso do smartphone para conexão social e entretenimento são os principais propósitos de uso do smartphone associados à DS.

\section{Outras dependências}

A DS também foi associada a outras formas de dependência. Dois estudos encontraram uma associação positiva e significativa entre a dependência de álcool e a DS ${ }^{10,24}$. Beison \& Rademacher ${ }^{24}$ evidenciaram que a história familiar de alcoolismo estava associada à DS. Os autores sugeriram que esse fato pode ocorrer devido a um componente genético para o comportamento compulsivo que favorece o desenvolvimento de muitas dependências, incluindo a DS (beta $=0,353, p<0,001$ ). Um estudo encontrou uma associação positiva entre a DS e a dependência de nicotina (mulheres: $\mathrm{OR}=2,151$, $p<0,05$; homens: $O R=2,467, p<0,001)^{53}$. Choi et al. ${ }^{10}$ encontraram uma associação entre a DS e a DI (beta = 0,184; p < 0,001), e Lee ${ }^{43}$ encontraram uma associação entre a DS e a dependência de Facebook (beta = 0,114; $p=0,043)$. Portanto, a DS está associada a outras formas de dependência, principalmente alcoolismo e dependências tecnológicas.

\section{Transtornos e sintomas psiquiátricos}

Alguns transtornos psiquiátricos foram associados à DS. A associação entre a DS e os transtornos de ansiedade foi encontrada em 11 estudos $9,18,23,25,27,31,33,37,42$. Os coeficientes betas variaram de 0,130 a 1,320.

A depressão também foi associada à DS em 10 estudos $17,18,20,25,29,33,39,45,51,55,58$. Os coeficientes betas variaram de 0,067 a 2,572. Além disso, um estudo desenvolvido por Yang et al., em $2010^{53}$, no sul do Taiwan, encontraram uma associação entre ideação suicida e DS (mulheres: $O R=1,835, p<0,001$; homens: $\mathrm{OR}=2,208, \mathrm{p}<0,001)$.

Sete estudos encontraram uma associação entre a DS e altos níveis de estresse $20,25,38,40,42,45,59$. Os coeficientes betas variaram de 0,18 a 0,74. Lee encontrou uma associação entre estresse tecnológico e DS (beta = 0,74; $p<0,001$ ), enquanto Augner \& Hacker ${ }^{20}$ encontrou uma associação entre a DS e o estresse crônico (beta = 0,27; $p<0,001)$. Além disso, Hong et al. ${ }^{27}$ encontrou uma associação entre a DS e o estresse familiar $(r=0,194 ; p$ $<0,001)$, o estresse emocional $(r=0,232 ; p<0,001)$ e o estresse acadêmico ( $z=-2,76 ; p<0,01)$.

Quatro estudos encontraram uma associação entre transtornos do sono e DS $18,36,53$. Todos foram realizados com estudantes universitários, e os coeficientes beta variaram de 0,329 a 1,870.

Dessa forma, os transtornos de ansiedade e a depressão foram os principais transtornos psiquiátricos associados à DS; enquanto estresse e insônia foram os principais sintomas psiquiátricos associados ao constructo.

\section{Traços de personalidade}

A DS também foi associada a traços de personalidade. Seis estudos encontraram uma associação com alta impulsividade ${ }^{9,17,29-32}$. Os coeficientes beta variaram de 0,04 a 0,38. No estudo de Billieux et al..$^{31}$, a DS associouse aos seguintes subdomínios da impulsividade: urgência $(\mathrm{OR}=1,05, \mathrm{p}=0,04)$ e falta de perseverança $(\mathrm{OR}=$ $1,07, p=0,04)$, enquanto não houve associação com os subdomínios: falta de premeditação $(O R=0,98, p=$ $0,59)$ e busca de sensação $(O R=1,02, p=0,31)$.

A baixa autoestima também foi associada à DS em quatro estudos $22,27,53,54$. Os coeficientes betas variaram de 0,15 a 0,239.

Três estudos encontraram que a extroversão estava associada à DS20,27,29. Os coeficientes betas variaram de 0,13 a 0,26 .

Dois estudos encontraram uma associação entre a DS e personalidade do tipo $A^{33,53}$. De acordo com Matar Boumosleh \& Jaalouk ${ }^{33}$, jovens com personalidade do tipo A que experimentam altos níveis de estresse e hipotimia podem ter mecanismos de enfrentamento deficientes e, portanto, maior suscetibilidade à DS (beta $=4,109, \mathrm{p}=$ 0,019). Dois estudos encontraram uma associação entre a DS e traço de personalidade agressiva ${ }^{17,53}$. No estudo de Yang et al. ${ }^{53}$, a razão de chances para mulheres foi de 1,870 ( $p<0,001)$ e para homens foi de 1,788 ( $p<$ 0,001); no estudo de Kim et al., o coeficiente beta foi de $0,020(p<0,001)^{17}$. 


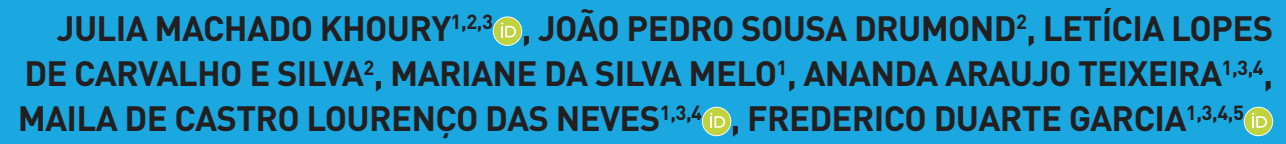

Departamento de Saúde Mental, Faculdade de Medicina de Minas Gerais, Belo Horizonte, MG Departamento de Clínica Médica, Faculdade de Saúde e Ecologia Humana, Vespasiano, MG. Programa de Pós-Graduação em Medicina Molecular, Faculdade de Medicina, Universidade Federal de Minas Gerais (UFMG), Belo Horizonte, MG. ${ }^{4}$ INCT de Medicina Molecular, UFMG, Belo Horizonte, MG. ${ }^{5}$ Unité Inserm U1073, Rouen, França.

Uma relação entre a DS e traço de personalidade materialista foi encontrada em apenas um estudo (beta $=0,38 ; p<0,01)^{46}$. Uma associação entre a DS e perfeccionismo foi encontrada em um estudo ${ }^{25}$. Os autores relataram que indivíduos perfeccionistas usam smartphones de forma abusiva para verificar se tudo está sob controle e para evitar riscos ou falhas, o que aumenta a chance de desenvolver a DS $(O R=1,15 ; p<0,001)$.

Um estudo sugeriu que indivíduos com traço de personalidade de neuroticismo têm mais chance de serem dependentes de smartphones ${ }^{19}$. Outro estudo sugeriu que a alta sensibilidade às recompensas está associada à DS (OR = 1,021; $p=0,009)$, o que pode estar relacionado ao fato de que o smartphone pode ser uma fonte de recompensas imediatas ${ }^{10}$.

O estudo de Lee et al. ${ }^{60}$ encontrou uma associação positiva entre a DS e o comportamento multitarefa (beta $=0,297 ; p<0,001$.

O estudo de Augner \& Hacker ${ }^{20}$ sugeriu que a baixa estabilidade emocional é um traço psicológico associado à DS (beta $=-0,21 ; p=0,002)$. Além disso, o estudo de Takao et al. ${ }^{21}$ concluiu que indivíduos com alto automonitoramento (beta $=0,22 ; p=0,001$ ) e indivíduos com necessidade de aprovação social (beta $=-0,17 ; p$ $=0,001$ ) têm mais chance de serem dependentes de smartphone.

Leung 22 conduziram, no Japão, um estudo com a intenção de investigar como alguns fatores demográficos e psicológicos estão relacionados à DS. Os resultados sugeriram que a alta tendência ao tédio (beta $=0,14 ; p<$ 0,01) e o traço de personalidade de busca de sensações (beta $=0,21 ; p<0,001$ ) possuem uma forte associação com a DS.

Portanto, os principais traços de personalidade associados à DS foram a alta impulsividade, a alta agressividade, a baixa autoestima, a extroversão, a personalidade do tipo A e a personalidade multitarefa (Tabela 1).

\section{Discussão}

Nesta revisão bibliográfica, analisamos 42 estudos que avaliaram os principais fatores associados à DS. A maioria dos estudos foi realizada nos países orientais, e as principais populações analisadas foram as dos estudantes universitários.
Os principais fatores sociodemográficos associados à DS foram sexo feminino, idade entre 18 e 25 anos e alta renda familiar mensal. Os principais hábitos de vida associados foram cronótipo noturno, baixa atividade física e sedentarismo. Os principais hábitos de vida associados à DS foram o uso do aparelho por mais de 4 horas por dia, a posição de carregar o smartphone em frente ao peito ou nos bolsos da calça, a idade do primeiro uso antes dos 13 anos e o tempo até o primeiro uso pela manhã até 10 minutos. Os principais conteúdos associados à DS foram as redes sociais. Os principais propósitos de uso associados à DS foram para conexão social, diversão e entretenimento. As principais síndromes de dependência associadas foram a dependência de álcool e as dependências tecnológicas. Os principais transtornos e/ou sintomas psiquiátricos associados foram os transtornos depressivos e ansiosos, o estresse e a insônia. Por fim, os principais traços de personalidade associados à DS foram alta impulsividade, alta agressividade, baixa autoestima, personalidade do tipo A e personalidade multitarefas.

A associação entre o sexo feminino e a DS pode acontecer por diversos motivos. Mulheres são mais propensas a utilizar e se tornar dependentes de redes sociais ${ }^{10,23,27,60,61-68}$, e as redes sociais são o principal conteúdo associado à DS10,60,69-74. Portanto, essa associação pode ser mediada pelo uso abusivo de redes sociais. Já os homens são mais propensos a serem dependentes de jogos eletrônicos ${ }^{75-77}$, os quais são menos adaptados aos smartphones do que a computadores de mesa, representando, assim, menor risco para a DS ${ }^{75-77}$. Outra hipótese para explicar a associação entre o sexo feminino e a DS é que as mulheres apresentam maior prevalência de transtornos ansiosos e depressivos ${ }^{78,79}$ e, portanto, podem ter maior chance de utilizar o smartphone como reforço negativo, o que aumenta a chance do desenvolvimento da dependência ao aparelho.

A idade jovem pode ser um fator de risco para o desenvolvimento da DS devido à imaturidade do córtex pré-frontal (PFC), típica de outras dependências químicas e comportamentais $69,70,80$. Essa imaturidade reduz o controle de impulsos e dificulta estratégias para controlar o uso de substâncias e a realização de comportamentos específicos ${ }^{80-82}$. É importante ressaltar que a DS pode contribuir para o baixo desempenho acadêmico dos 


\section{ARTIGO DE REVISÃO}

\section{JULIA MACHADO KHOURY \\ JOÃO PEDRO SOUSA DRUMOND \\ LETÍCIA LOPES DE CARVALHO E SILVA \\ MARIANE DA SILVA MELO \\ ANANDA ARAUJO TEIXEIRA \\ MAILA DE CASTRO LOURENCCO DAS NEVES \\ FREDERICO DUARTE GARCIA}

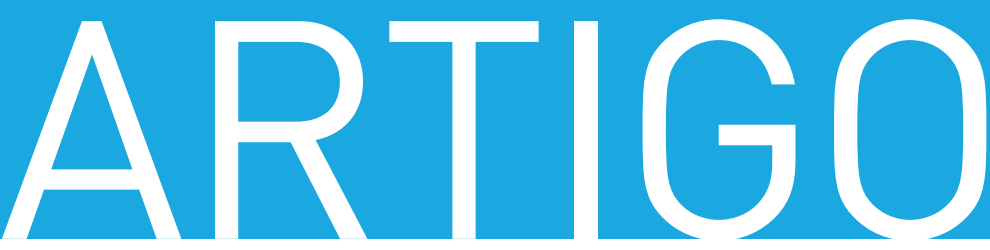

estudantes $1,19,46,83$, o que indica a necessidade de mecanismos para prevenir o uso abusivo de smartphones por essa população.

A associação entre a DS e a alta renda familiar mensal pode estar relacionada ao maior acesso aos dispositivos por pessoas com renda mais alta. Sendo assim, ser de uma classe socioeconômica mais alta aumenta a probabilidade de ter e usar smartphones por um período de tempo maior, uma vez que esses indivíduos podem arcar com gastos maiores com acesso à internet ${ }^{54,62,84,85}$. Como foi demonstrado para as dependências químicas e comportamentais, os tempos mais longos gastos com uma substância ou a execução de um comportamento específico são fatores de risco para o desenvolvimento das síndromes de dependência15,86,87.

A associação entre a DS e o cronótipo noturno pode ocorrer devido ao fato de pessoas com hábitos noturnos poderem fazer uso abusivo do smartphone como forma de combater o tédio, que é mais frequente à noite. O hábito de usar o smartphone à noite também pode prejudicar o sono e causar insônia18,23,36,53, o que pode ajudar os indivíduos a ficarem acordados por mais tempo à noite fazendo uso do aparelho. Portanto, o cronótipo noturno pode ser causa e/ou consequência da DS.

O nível reduzido de atividade física e o sedentarismo também podem ser causa e/ou consequência da DS. Indivíduos que não gostam de se exercitar tendem a se concentrar mais em atividades intelectuais e estão mais familiarizados com a tecnologia ${ }^{88}$, o que pode favorecer o desenvolvimento da DS. Por outro lado, o uso abusivo do smartphone pode favorecer o estilo de vida sedentário, uma vez que indivíduos dependentes tendem a restringir o repertório e abandonar atividades incompatíveis com o seu uso ${ }^{12,89}$.

Os hábitos de uso do smartphone que estão associados à DS são similares aos hábitos de uso de drogas e de realização de comportamentos que já foram associados às dependências químicas e comportamentais ${ }^{90,91}$. O uso por um período maior de tempo, o início precoce do uso e o uso em um curto período de tempo após o despertar são comportamentos relacionados à dependência de substâncias psicoativas, à dependência de jogos e a outras síndromes de dependência9,90. Portanto, essa pode ser mais uma evidência empírica para a caracterização da DS como uma síndrome de dependência.

A associação entre a DS e a dependência de redes sociais pode acontecer por alguns motivos. A portabilidade e a acessibilidade do smartphone favorecem o acesso frequente e rápido às redes sociais ${ }^{11,60,72,92}$. Nesse sentido, quando comparado ao acesso às redes sociais por meio de computadores de mesa, os smartphones estariam associados a um maior risco de dependência93,94. Tais como drogas com meias-vidas mais curtas e pico plasmático mais rápido, as curtidas e os comentários nas redes sociais poderiam liberar pulsos fásicos de dopamina no núcleo accumbens, o que geraria intenso reforço positivo, possivelmente favorecendo o uso e dependência aos smartphones ${ }^{95}$. Essa é a principal teoria que explica a maior dependência do crack em comparação à cocaína inalada96,97, bem como a maior dependência dos benzodiazepínicos de meia-vida curta quando comparados àqueles com meiavida mais longa98,99. As redes sociais são mais adaptadas aos smartphones, uma vez que os aparelhos permitem o acesso em qualquer lugar e a qualquer momento, o que facilita a interação virtual em tempo real e incentiva os rituais de checagem regular ${ }^{87,100}$. As redes sociais também podem ser usadas como reforço negativo, reduzindo sentimentos ansiosos e depressivos como uma espécie de fuga da vida real e como uma forma de procrastinação em indivíduos com dificuldade de concentração. A associação entre o uso de redes sociais e a DS também pode explicar a associação entre o propósito de usar o smartphone e a DS, já que a principal motivação de usar smartphone pelos dependentes do aparelho é para conexão social e entretenimento ${ }^{34}$.

A associação entre a dependência de álcool, as dependências tecnológicas e a DS pode ocorrer principalmente devido à dificuldade de controle dos impulsos e à maior suscetibilidade a comportamentos compulsivos que esses indivíduos apresentam $3,10,74,101$. Portanto, é possível que exista um perfil de vulnerabilidade que predisponha os indivíduos ao desenvolvimento das síndromes de dependência.

A associação entre sintomas/transtornos psiquiátricos e a DS pode ocorrer porque os indivíduos abusam do smartphone para aliviar sentimentos negativos ou para fugir da vida real. Essa forma de reforço negativo pode contribuir para o uso compulsivo e o desenvolvimento da dependência. Por outro lado, a DS pode contribuir para o desenvolvimento ou para o aumento dos sintomas e transtornos psiquiátricos, uma vez que aumenta o isolamento social e prejudica o sono ${ }^{18,23,36,53}$. Portanto, sintomas e transtornos psiquiátricos podem ser causa e/ ou consequência da DS. 


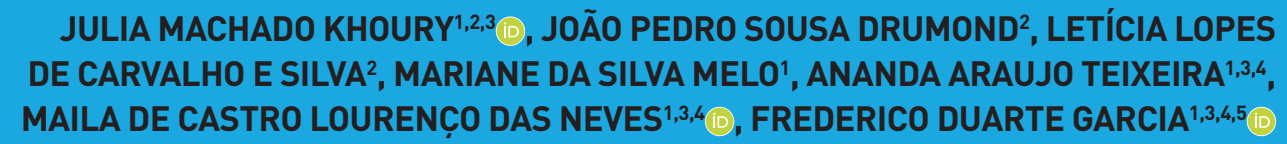

1 Departamento de Saúde Mental, Faculdade de Medicina de Minas Gerais, Belo Horizonte, MG. Departamento de Clínica Médica, Faculdade de Saúde e Ecologia Humana, Vespasiano, MG. Programa de Pós-Graduação em Medicina Molecular, Faculdade de Medicina, Universidade Federal de Minas Gerais (UFMG), Belo Horizonte, MG. ${ }^{4}$ INCT de Medicina Molecular, UFMG, Belo Horizonte, MG. ${ }^{5}$ Unité Inserm U1073, Rouen, França.

A DS pode estar associada a alguns traços de personalidade como fator predisponente ou como consequência. Indivíduos com alta impulsividade podem ter maior dificuldade em controlar o desejo de checar regularmente o smartphone, o que pode favorecer o desenvolvimento da dependência. A baixa autoestima pode favorecer o abuso de smartphones, já que os indivíduos são mais sensíveis a curtidas e comentários nas redes sociais ${ }^{100}$, o que pode estimular o ritual de checagem regular e, consequentemente, a DS. As personalidades do tipo A e multitarefa podem estar associadas à DS devido ao medo de perder informações transmitidas através do aparelho (fear of missing out FOMO), o que contribui para o ritual de checagem regular e, consequentemente, para a DS ${ }^{34}$.

\section{CONCLUSÃO}

Mulheres jovens, com alta renda familiar mensal, cronótipo noturno e estilo de vida sedentário, que abusam de redes sociais e fazem uso dos smartphones por mais de 4 horas por dia são mais propensas a se tornarem dependentes de smartphone. Além disso, indivíduos com comorbidades psiquiátricas e outras síndromes de dependência também têm maior chance de desenvolver a DS. O estudo dos fatores associados facilita a identificação dos indivíduos com maior probabilidade de desenvolver a DS e, consequentemente, o posterior desenvolvimento de estratégias preventivas e terapêuticas específicas e direcionadas. Além disso, - estudo desses indivíduos aumenta a probabilidade de que a DS seja reconhecida como um transtorno psiquiátrico.

\section{Agradecimentos}

Este trabalho foi financiado pela Fundação de Amparo à Pesquisa do Estado de Minas Gerais (FAPEMIG; processos APQ-02572-16 e APQ-04347-17) e pelo Conselho Nacional de Desenvolvimento Científico e Tecnológico (CNPq; 313944/2018-0).

Artigo submetido em 25/05/2019, aceito em 27/05/2019. Os autores informam não haver conflitos de interesse associados à publicação deste artigo.

Fontes de financiamento inexistentes.

Correspondência: Frederico Duarte Garcia, Departamento de Saúde Mental, Universidade Federal de Minas Gerais, Av. Professor Alfredo Balena, 190, sala
240, CEP 30130-100, Belo Horizonte, MG. Tel.: +55 31 3409.9786. E-mail: frederico.garciad@gmail.com

\section{Referências}

1. Head M, Ziolkowski N. Understanding student attitudes of mobile phone features: rethinking adoption through conjoint, cluster and SEM analyses. Comput Human Behav. 2012:28:2331-9.

2. Shambare R, Rugimbana R, Zhowa T. Are mobile phones the 21st century addiction? Afri J Bus Manage. 2012;6:573-7.

3. Al-Barashdi HS, Bouazza A, Jabur NH. Smartphone addiction among university undergraduates: a literature review. J Sci Res Rep. 2015;4:210-25.

4. Kubey RW, Lavin MJ, Barrows JR. Internet use and collegiate academic performance decrements: early findings. J Commun. 2001;51:366-82.

5. Palen L, Salzman M, Youngs E. Discovery and integration of mobile communications in everyday life. Pers Ubiquitous Comput. 2001;5:109-22.

6. Lee KC, Perry SD. Student instant message use in a ubiquitous computing environment: effects of deficient self-regulation. J Broadcast Electron Media. 2004:48:399-420.

7. Klauer S, Guo F, Simons-Morton B, Ouimet MC. Distracted driving and risk of road crashes among novice and experienced drivers. N Engl J Med. 2014;370:54-9.

8. Ahmed I, Qazi TF, Perji KA. Mobile phone to youngsters: necessity or addiction. Afri J Bus Manage. 2011;5:12512-9.

9. Choi J, Rho MJ, Kim Y, Yook IH, Yu H, Kim DJ, et al. Smartphonedependenceclassificationusingtensor factorization. PLoS One. 2017;12:e0177629.

10. Choi SW, Kim DJ, Choi JS, Ahn H, Choi EJ, Song WY, et al. Comparison of risk and protective factors associated with smartphone addiction and Internet addiction. J Behav Addict. 2015;4:30814.

11. Chóliz M. Mobile phone addiction: a point of issue. Addiction. 2010;105:373-4.

12. Kwon M, Lee JY, Won WY, Park JW, Min JA, Hahn C, et al. Development and validation of a smartphone addiction scale (SAS). PloS One. 2013;8:e56936. 
13. Billieux J, Maurage P, Lopez-Fernandez $O$, Kuss DJ, Griffiths MD. Can disordered mobile phone use be considered a behavioral addiction? An update on current evidence and a comprehensive model for future research. Curr Addict Rep. 2015;2:15662.

14. de Oliveira AFP. Neurobiologia dos comportamentos aditivos [thesis]. Porto: Universidade do Porto; 2012.

15. Goodman A. Addiction: definition and implications. Br J Addict. 1990;85:1403-8.

16. Randler C, Wolfgang L, Matt K, Demirhan E, Horzum MB, Beşoluk S. Smartphone addiction proneness in relation to sleep and morningnesseveningness in German adolescents. J Behav Addict. 2016;5:465-73.

17. Kim SE, Kim JW, Jee YS. Relationship between smartphone addiction and physical activity in Chinese international students in Korea. J Behav Addict. 2015;4:200-5.

18. Demirci K, Akgonul M, Akpinar A. Relationship of smartphone use severity with sleep quality, depression, and anxiety in university students. J Behav Addict. 2015;4:85-92.

19. Mok JY, Choi SW, Kim DJ, Choi JS, Lee J, Ahn $\mathrm{H}$, et al. Latent class analysis on internet and smartphone addiction in college students. Neuropsychiatr Dis Treat. 2014;10:817-28.

20. Augner C, Hacker GW. Associations between problematic mobile phone use and psychological parameters in young adults. Int J Public Health. 2012;57:437-41.

21. Takao M, Takahashi S, Kitamura M. Addictive personality and problematic mobile phone use. Cyberpsychol Behav. 2009;12:501-7.

22. Leung L. Linking psychological attributes to addiction and improper use of the mobile phone among adolescents in Hong Kong. J Child Media. 2008;2:93-113.

23. Jenaro C, Flores N, Gómez-Vela M, GonzálezGil F, Caballo C. Problematic internet and cellphone use: psychological, bahavioral, and health correlates. Addict Res Theory. 2007;15:309-20.

24. Beison A, Rademacher DJ. Relationship between family history of alcohol addiction, parents' education level, and smartphone problem use scale scores. J Behav Addict. 2017;6:84-91.

25. Long J, Liu TQ, Liao YH, Qi C, He HY, Chen SB, et al. Prevalence and correlates of problematic smartphone use in a large random sample of Chinese undergraduates. BMC Psychiatry. 2016;16:408.

26. Lee KE, Kim SH, Ha TY, Yoo YM, Han JJ, Jung $\mathrm{JH}$, et al. Dependency on smartphone use and its association with anxiety in Korea. Public Health Rep. 2016;131:411-9.

27. Hong FY, Chiu SI, Huang DH. A model of the relationshipbetween psychologicalcharacteristics, mobile phone addiction and use of mobile phones by Taiwanese university female students. Comput Human Behav. 2012;28:2152-9.

28. Kim Y, Jeong JE, Cho H, Jung DJ, Kwak M, Rho MJ, et al. Personality factors predicting smartphone addiction predisposition: behavioral inhibition and activation systems, impulsivity, and self-control. PLoS One. 2016;11:e0159788.

29. Smetaniuk P. A preliminary investigation into the prevalence and prediction of problematic cell phone use. J Behav Addict. 2014;3:41-53.

30. Wu AM, Cheung VI, Ku L, Hung EP. Psychological risk factors of addiction to social networking sites among Chinese smartphone users. J Behav Addict. 2013;2:160-6.

31. Billieux J, Van der Linden $M$, d'Acremont $M$, Ceschi G, Zermatten A. Does impulsivity relate to perceived dependence on and actual use of the mobile phone? Appl Cogn Psychol. 2007;21:52737.

32. Billieux J, Van der Linden M, Rochat L. The role of impulsivity in actual and problematic use of the mobile phone. Appl Cogn Psychol. 2008;22:1195210.

33. Matar Boumosleh J, Jaalouk D. Depression, anxiety, and smartphone addiction in university students- a cross sectional study. PLoS One. 2017;12:e0182239.

34. Oberst U, Wegmann E, Stodt B, Brand M, Chamarro A. Negative consequences from heavy social networking in adolescents: the mediating role of fear of missing out. J Adolesc. 2017;55:5160. 


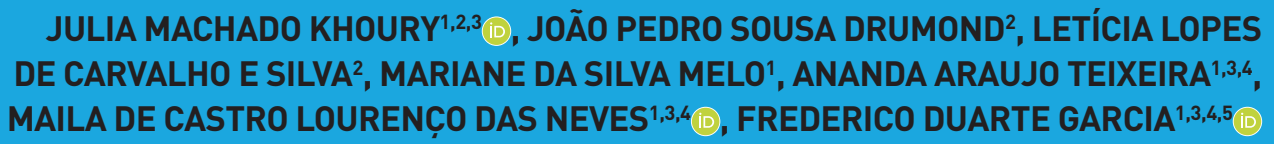

Departamento de Saúde Mental, Faculdade de Medicina de Minas Gerais, Belo Horizonte, MG. Departamento de Clínica Médica, Faculdade de Saúde e Ecologia Humana, Vespasiano, MG. Programa de Pós-Graduação em Medicina Molecular, Faculdade de Medicina, Universidade Federal de Minas Gerais (UFMG), Belo Horizonte, MG. ${ }^{4}$ INCT de Medicina Molecular, UFMG, Belo Horizonte, MG. ${ }^{5}$ Unité Inserm U1073, Rouen, França.

35. Randler C, Wolfgang L, Matt K, Demirhan E, Horzum MB, Beşoluk S. Smartphone addiction proneness in relation to sleep and morningnesseveningness in German adolescents. J Behav Addict. 2016:5:465-73.

36. Alosaimi FD, Alyahya $\mathrm{H}$, Alshahwan $\mathrm{H}$, Al Mahyijari N, Shaik SA. Smartphone addiction among university students in Riyadh, Saudi Arabia. Saudi Med J. 2016:37:675-83.

37. Darcin AE, Kose S, Noyan CO, Nurmedov S, Yılmaz O, Dilbaz N. Smartphone addiction and its relationship with social anxiety and loneliness. Behav Inf Technol. 2016;35:520-5.

38. Haug S, Castro RP, Kwon M, Filler A, Kowatsch T, Schaub MP. Smartphone use and smartphone addiction among young people in Switzerland. J Behav Addict. 2015;4:299-307.

39. Toda M, Nishio N, Takeshita T. Predictive factors for smartphone dependence: relationship to demographic characteristics, chronotype, and depressive state of university students. Open J Prev Med. 2015;5:456-62.

40. Wang JL, Wang HZ, Gaskin J, Wang LH. The role of stress and motivation in problematic smartphone use among college students. Comput Human Behav. 2015;53:181-8.

41. Lin $Y H$, Lin $Y C$, Lee $Y H$, Lin $P H$, Lin SH, Chang LR, et al. Time distortion associated with smartphone addiction: identifying smartphone addiction via a mobile application (App). J Psychiatr Res. 2015;65:139-45

42. Bian M, Leung L. Smartphone addiction: linking loneliness, shyness, symptoms and patterns of use to social capital. Media Asia. 2014;41:159-76.

43. Lee EB. Too much information: heavy smartphone and Facebook utilization by African American young adults. J Black Stud. 2015;46:44-61.

44. Chiu SI. The relationship between life stress and smartphone addiction on Taiwanese university student: a mediation model of learning selfefficacy and social self-efficacy. Comput Human Behav. 2014:34:49-57.

45. Harwood J, Dooley JJ, Scott AJ, Joiner R. Constantly connected-the effects of smartdevices on mental health. Comput Human Behav. 2014;34:267-72.
46. Lee YK, Chang CT, Lin Y, Cheng ZH. The dark side of smartphone usage: psychological traits, compulsive behavior and technostress. Comput Human Behav. 2014;31:373-83.

47. Cagan O, Unsal A, Celik N. Evaluation of college students' level of addiction to cellular phone and investigation on the relationship between the addiction and the level of depression. Procedia Soc Behav Sci. 2014;114:831-9.

48. Zheng F, Gao P, He M, Li M, Wang C, Zeng Q, et al. Association between mobile phone use and inattention in 7102 Chinese adolescents: a population-based cross-sectional study. BMC Public Health. 2014;14:1022.

49. Roberts JA, Yaya LH, Manolis C. The invisible addiction: cell-phone activities and addiction among male and female college students. J Behav Addict. 2014:3:254-65.

50. Sahin S, Ozdemir K, Unsal A, Temiz N. Evaluation of mobile phone addiction level and sleep quality in university students. Pak J Med Sci. 2013;29:913-8.

51. LuX, Watanabe J, Liu Q, Uji M, Shono M, Kitamura T. Internet and mobile phone text-messaging dependency: factor structure and correlation with dysphoric mood among Japanese adults. Comput Human Behav. 2011;27:1702-9.

52. Walsh SP, White KM, Cox S, McD Young R. Keeping in constant touch: the predictors of young Australians' mobile phone involvement. Comput Human Behav. 2011;27:333-42.

53. Yang YS, Yen JY, Ko CH, Cheng CP, Yen CF. The association between problematic cellular phone use and risky behaviors and low self-esteem among Taiwanese adolescents. BMC Public Health. 2010;10:217.

54. Zulkefly SN, Baharudin R. Mobile phone use amongst students in a university in Malaysia: its correlates and relationship to psychological health. Eur J Sci Res. 2009:37:206-18.

55. Yen CF, Tang TC, Yen JY, Lin HC, Huang CF, Liu SC, et al. Symptoms of problematic cellular phone use, functional impairment and its association with depression among adolescents in Southern Taiwan. J Adolesc. 2009;32:863-73. 
JULIA MACHADO KHOURY

JOÃO PEDRO SOUSA DRUMOND

LETÍCIA LOPES DE CARVALHO E SILVA

MARIANE DA SILVA MELO

ANANDA ARAUJO TEIXEIRA

MAILA DE CASTRO LOURENÇO DAS NEVES

FREDERICO DUARTE GARCIA
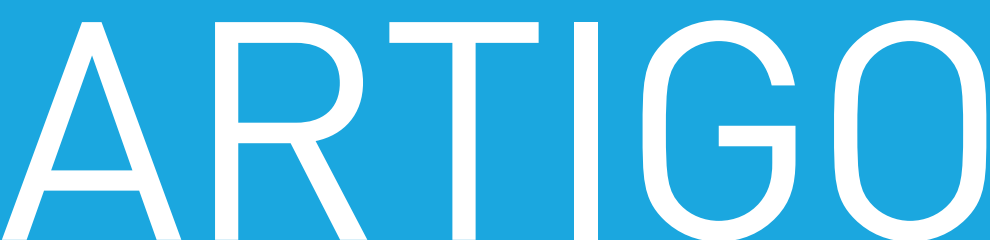

56. Bianchi A, Phillips JG. Psychological predictors of problem mobile phone use. Cyberpsychol Behav. 2005;8:39-51.

57. Toda M, Monden K, Kubo K, Morimoto K. Mobile phone dependence and health-related lifestyle of university students. Soc Behav Pers. 2006;34:1277-84.

58. da Luz FQ, Sainsbury A, Mannan H, Touyz S, Mitchison D, Girosi F, et al. An investigation of relationships between disordered eating behaviors, weight/shape overvaluation and mood in the general population. Appetite. 2018;129:1924.

59. Hong FY, Huang DH, Lin DY, Chiu SL. Analysis of the psychological traits, Facebook usage, and Facebook addiction model of Taiwanese university students. Telematics Inform. 2014;31:597-606.

60. Lee H, Kim JW, Choi TY. Risk factors for smartphone addiction in Korean adolescents: smartphone use patterns. J Korean Med Sci. 2017;32:1674-9.

61. Martinotti G, Villella C, Di Thiene D, Di Nicola M, Bria P, Conte G, et al. Problematic mobile phone use in adolescence: a cross sectional study. J Public Health. 2011;19:545-51.

62. Abojedy A. Mobile phone addiction and its relationship with self-discloser among sample of students from University of Jordan and Amman Al-Ahliyya University. Jordan J Educ Sci. 2008:4:137-50.

63. Devis-Devis J, Peiró-Velert C, Beltrán-Carrillo VJ, Tomás JM. Screen media time usage of 12-16 year-old Spanish school adolescents: effects of personal and socioeconomic factors, season and type of day. J Adolesc. 2009;32:213-31.

64. Chóliz M. Mobile-phone addiction in adolescence: the test of mobile phone dependence (TMD). Prog Health Sci. 2012;2:33-44.

65. Sánchez-Martínez M, Otero A. Factors associated with cell phone use in adolescents in the community of Madrid (Spain). Cyberpsychol Behav. 2009;12:131-7.

66. Kawasaki N, Tanei S, Ogata F, Burapadaja S, Loetkham C, Nakamura T, et al. Survey on cellular phone usage on students in Thailand. J Physiol Anthropol. 2006;25:377-82.
67. Chung N. Korean adolescent girls' addictive use of mobile phones to maintain interpersonal solidarity. Soc Behav Pers. 2011;39:1349-58.

68. Hakoama M, Hakoyama S. The impact of cell phone use on social networking and development among college students. AABSS J. 2011;15:1-20.

69. Igarashi T, Motoyoshi T, Takai J, Yoshida T. No mobile, no life: self-perception and text-message dependency among Japanese high school students. Comput Human Behav. 2008;24:231124.

70. Weiser EB. Gender differences in Internet use patterns and Internet application preferences: a two-sample comparison. Cyberpsychol Behav. 2000;3:167-77.

71. Heo J, Oh J, Subramanian SV, Kim Y, Kawachi I. Addictive internet use among Korean adolescents: a national survey. PloS One. 2014;9:e87819.

72. Jeong SH, Kim H, Yum JY, Hwang Y. What type of content are smartphone users addicted to? SNS vs. games. Comput Human Behav. 2016;54:10-7.

73. Kneer J, Glock S. Escaping in digital games: the relationship between playing motives and addictive tendencies in males. Comput Human Behav. 2013;29:1415-20.

74. Milosevic J, Žeželj I. Psychological predictors of addictive social networking sites use: the case of Serbia. Comput Human Behav. 2014;32:229-34.

75. Fattore L, Melis M, Fadda P, Fratta W. Sex differences in addictive disorders. Front Neuroendocrinol. 2014;35:272-84.

76. Cooper A, Morahan-Martin J, Mathy RM, Maheu M. Toward an increased understanding of user demographics in online sexual activities. J Sex Marital Ther. 2002;28:105-29.

77. Johansson A, Götestam KG. Internet addiction: characteristics of a questionnaire and prevalence in Norwegian youth (12-18 years). Scand J Psychol. 2004;45:223-9.

78. Nolen-Hoeksema S. Gender differences in depression. Curr Dir Psychol Sci. 2001;10:173-6.

79. Taylor M, Jinabhai CC, Naidoo K, Kleinschmidt I, Dlamini SB. An epidemiological perspective of substance use among high school pupils in rural KwaZulu-Natal S Afr Med J. 2003;93:136-40. 


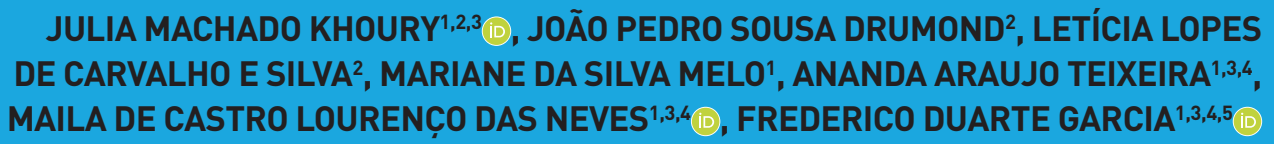

Departamento de Saúde Mental, Faculdade de Medicina de Minas Gerais, Belo Horizonte, MG. Departamento de Clínica Médica, Faculdade de Saúde e Ecologia Humana, Vespasiano, MG. Programa de Pós-Graduação em Medicina Molecular, Faculdade de Medicina, Universidade Federal de Minas Gerais (UFMG), Belo Horizonte, MG. ${ }^{4}$ INCT de Medicina Molecular, UFMG, Belo Horizonte, MG. ${ }^{5}$ Unité Inserm U1073, Rouen, França.

80. Bechara A, Dolan S, Denburg N, Hindes A, Anderson SW, Nathan PE. Decision-making deficits, linked to a dysfunctional ventromedial prefrontal cortex, revealed in alcohol and stimulant abusers. Neuropsychologia. 2001;39:376-89.

81. Bowden-Jones $H$, McPhillips $M$, Rogers $R$, Hutton S, Joyce E. Risk-taking on tests sensitive to ventromedial prefrontal cortex dysfunction predicts early relapse in alcohol dependency: a pilot study. J Neuropsychiatry Clin Neurosci. 2005:17:417-20.

82. Damasio AR. The somatic marker hypothesis and the possible functions of the prefrontal cortex. Philos Trans R Soc Lond B Biol Sci. 1996;351:141320.

83. Kim HJ, Min JY, Kim HJ, Min KB. Association between psychological and self-assessed health status and smartphone overuse among Korean college students. J Ment Health. 2019;28:11-6.

84. Prezza M, Pacilli MG, Dinelli S. Loneliness and new technologies in a group of Roman students. Comput Human Behav. 2004:20:691-709.

85. Castells M, Fernandez-Ardevol M, Qiu JL, Sey A. The mobile communication society: a crosscultural analysis of available evidence on the social uses of wireless communication technology [Interntet]. 2004 Oct [cited 2020 Aug 28]. citeseerx.ist.psu.edu/viewdoc/download?doi=10. 1.1.109.3872\&rep=rep1\&type $=$ pdf

86. Holden C. 'Behavioral' addictions: do they exist? Science. 2001;294:980-2.

87. Brewer JA, Potenza MN. The neurobiology and genetics of impulse control disorders: relationships to drug addictions. Biochem Pharmacol. 2008:75:63-75.

88. Wadsworth D, Gleason M, Stoner L. Can sedentary behaviour be considered a cultural maladaptation? Perspect Public Health. 2014;134:20-1.

89. Lin YH, Chang LR, Lee YH, Tseng HW, Kuo TB, Chen $\mathrm{SH}$. Development and validation of the smartphone addiction inventory (SPAI). PLoS One. 2014;9:e98312.

90. O'Brien C. Addiction and dependence in DSM-V. Addiction. 2011;106:866-7.
91. Associação Americana de Psiquiatria. Manual Diagnóstico e Estatístico de Transtornos Mentais, $5^{a}$ edição (DSM-5). Porto Alegre: Artmed; 2014.

92. Granda P, Jimena L. ¿Se constituye el uso del Smartphone en una adicción? [Internet]. 2013 [cited 2020 Aug 28]. pt.scribd.com/ document/328955593/Se-Constituye-El-UsoDel-Smartphone-en-Una-Adiccion

93. Koob GF, Volkow ND. Neurobiology of addiction: a neurocircuitry analysis. Lancet Psychiatry, 2016. 3(8): p. 760-73.

94. Holly EN, Boyson CO, Montagud-Romero S, Stein DJ, Gobrogge KL, DeBold JF, et al. Episodic social stress-escalated cocaine self-administration: role of phasic and tonic corticotropin releasing factor in the anterior and posterior ventral tegmental area. J Neurosci. 2016:36:4093-105.

95. Pascoli V, Terrier J, Hiver A, Lüscher C. Sufficiency of mesolimbic dopamine neuron stimulation for the progression to addiction. Neuron. 2015;88:105466.

96. Gawin FH. Cocaine addiction: psychology and neurophysiology. Science. 1991;251:1580-6.

97. Gold MS, Miller NS, Jonas JM. Cocaine (and crack): neurobiology. In: Lowinson JH, Ruiz P, Millman RB, Langrod JG, editors. Substance abuse: a comprehensive textbook. 2nd ed. Baltimore: Williams \& Wilkins; 1992. p. 222-35.

98. Greenblatt DJ, Shader RI. Dependence, tolerance, and addiction to benzodiazepines: clinical and pharmacokinetic considerations. Drug Metab Rev. 1978;8:13-28

99. Smith DE, Wesson DR, Landry MJ. The pharmacology of benzodiazepine addiction. Fam Pract Recert. 1989;11(9 Suppl):94-107.

100. Ryan T, Chester A, Reece J, Xenos S. The uses and abuses of Facebook: a review of Facebook addiction. J Behav Addict. 2014;3:133-48.

101. Kuss DJ, Lopez-Fernandez O. Internet addiction and problematic Internet use: a systematic review of clinical research. World J Psychiatry. 2016;6:143-76. 